\title{
Selecting Project Delivery Systems Based on Simplified Neutrosophic Linguistic Preference Relations
}

\author{
Sui-Zhi Luo ${ }^{1}$, Peng-Fei Cheng ${ }^{2, *}$, Jian-Qiang Wang ${ }^{1}$ and Yuan-Ji Huang ${ }^{3,4}$ \\ 1 School of Business, Central South University, Changsha 410083, China; szlluo@csu.edu.cn (S.-Z.L.); \\ jqwang@csu.edu.cn (J.-Q.W.) \\ 2 School of Business, Hunan University of Science and Technology, Xiangtan 411201, China \\ 3 School of Economics and Management, Hunan University of Science and Engineering, Yongzhou 425199, \\ China; hs326@126.com \\ 4 Scientific Research and Organization Department of Hunan Federation of Social Science Circles, \\ Changsha 410003, China \\ * Correspondence: 1180033@hnust.edu.cn
}

Received: 14 July 2017; Accepted: 3 August 2017; Published: 9 August 2017

\begin{abstract}
Project delivery system selection is an essential part of project management. In the process of choosing appropriate transaction model, many factors should be under consideration, such as the capability and experience of proprietors, project implementation risk, and so on. How to make their comprehensive evaluations and select the optimal delivery system? This paper proposes a decision-making approach based on an extended linguistic preference structure: simplified neutrosophic linguistic preference relations (SNLPRs). The basic elements in SNLPRs are simplified neutrosophic linguistic numbers (SNLNs). First, several distance measures of SNLNs are introduced. A distance-based consistency index is provided to measure the consistency degree of a simplified neutrosophic linguistic preference relation (SNLPR). When the SNLPR is not acceptably consistent, a consistency-improving automatic iterative algorithm may be used. Afterwards, a decision-making method with SNLPRs is developed. The example of its application in project delivery systems' selection is offered, and a comparison analysis is given in the end as well.
\end{abstract}

Keywords: project delivery system selection; preference relations; simplified neutrosophic linguistic number; distance-based consistency index; improving consistency

\section{Introduction}

Construction is not only a carrier of fixed asset investment in a country, but also a channel to adjust products and industrial structures $[1,2]$. The choice of a project delivery system may be one of the most crucial elements of a project. There are multiple trading models that can be chosen. According to the complexity of projects and the relationships of owners with contractors, the delivery systems can be divided into four categories [3]. The general contract mode, which is the fixed price contract, mainly includes the Design Build (DB), Engineer Procure Construct Tumkey, and Design Build Operate (DBO). The management contract mode, namely the cost plus contract, principally contains the Construction-Management (CM) and Project-Management Contracting. The traditional trading model, called Design Bid Build (DBB), carries out the unit price contract. Others comprise Private Participating Infrastructure, Build Operate Transfer, Private Finance Initiative, and so on.

A lot of aspects, such as projects' characteristics, construction environment, owners' capacity, and market conditions, need to be decided in the system selection process [4]. Nevertheless, the choice of transaction modes is usually based on the subjective consciousness of the decision makers (DMs) 
in engineering practice. Only a few scientific and rational decision-making methods related to the selection of project delivery systems have been established. For instance, a fuzzy approach to pick out appropriate transaction systems was presented by Mostafavi and Karamouz [5]. Wang et al. [6] constructed a project delivery system model based on fuzzy sets. After that, the analytical hierarchy process (AHP) for choosing the trade model was provided as well $[7,8]$.

Neutrosophic sets (NSs), as a generalization of intuitionistic fuzzy sets, were originally proposed by Smarandache [9]. They can deal with consistent, hesitant, and inconsistent information at the same time. Figure 1 in Chen and Ye [10] shows the flow chart extended from fuzzy sets to neutrosophic sets (as well as simplified neutrosophic sets, single-valued neutrosophic sets, and interval neutrosophic sets). There are many extensions of NSs [11-15]. Numerous multi-criteria decision-making methods based on NSs and their extensions have been studied. For example, the ELECTRE approaches of NSs and interval neutrosophic sets (INSs) were presented by Peng et al. [16] and Zhang et al. [17], respectively. The extended TODIM [18] and MULTIMOORA [19] methods with NSs were also offered. Peng et al. [20] put forward a likelihood-based QUALIFLEX method of multi-valued neutrosophic numbers. Besides, the VIKOR method in line with INSs was developed by Bausys and Zavadskas [21]. Pouresmaeil et al. [22] proposed an extended TOPSIS and VIKOR approaches based on NSs. Furthermore, other approaches, such as the heronian mean operators [23], correlation coefficients [24,25], the WASPAS method [26-29], and the COPRAS $[30,31]$ method were discussed and applied in diverse areas.

However, no matter which of the methods mentioned above is used, DMs are asked to give their evaluation values directly. It may be not easy for them in some cases. Sometimes, people may be accustomed to make a judgment through comparing each pair of delivery systems, especially when they cannot make direct evaluations for each single model [32]. Hence, the decision-making methods based on a judgment matrix (preference relations) with NSs or other extensions may be valuable and necessary.

In general, there are two main types of preference relations. One expresses quantitative data, such as reciprocal preference relations [33,34], interval fuzzy preference relations [35-37], intuitionistic fuzzy preference relations $[38,39]$ triangular fuzzy preference relations $[40,41]$, hesitant fuzzy preference relations [42,43], and some extensions [44-46]. Another contains qualitative information, like linguistic preference relations (LPRs) [47-51], hesitant fuzzy linguistic preference relations (HFLPRs) [52-54], intuitionistic linguistic preference relations (ILPRs) [55], and so on [56-58].

This paper introduces a new type of preference relations, simplified neutrosophic linguistic preference relations (SNLPRs). The basic element in SNLPRs is a simplified neutrosophic linguistic number (SNLN). The aims of the paper are as follows. On the one hand, a matrix with qualitative information may be more suitable for selecting project delivery systems as many qualitative factors are considered such as owners' ability, the technical difficulty of the project, the uncertainty of the external environment, and so on. On the other hand, the aforementioned preference relations cannot describe DMs' degrees of certainty, hesitation, and negation of their qualitative judgment simultaneously. There is a hypothesis that the membership degree of linguistic values is 1 in LPRs and HFLPRs. ILPRs only express the consistency and inconsistency of linguistic values.

On the basis of linguistic term sets and simplified neutrosophic numbers [59], simplified neutrosophic linguistic numbers (SNLNs) may be one of the most widely used extensions [60-62]. The former stands for the qualitative evaluation values of project systems [63-65], and the latter are the truth-membership, indeterminacy-membership, and false-membership of the qualitative assessment. Most importantly, these degrees are independent of each other. Consequently, considering the fuzziness of human thought and the complexity of reality, expressing preference in terms of SNLNs may be more suitable.

This paper studies a decision-making method with preference relations under simplified neutrosophic linguistic environment. The following are our innovations:

(1) Propose the Hamming distance, Euclidean distance, and Hausdorff distance of two SNLNs. In addition, several relevant properties are discussed. 
(2) Present a new concept, SNLPRs. Subsequently, a distance-based consistency index is introduced to measure the consistency degree of SNLPRs.

(3) Develop a consistency-improving algorithm and a ranking method based on aggregation operators. A decision-making approach based on SNLPRs is described as well.

(4) Apply the proposed method to the project transaction model selection process. The practicability and effectiveness are demonstrated in a comparison analysis.

The remains of this paper are arranged as follows. Basic theories about SNLNs and LPRs are introduced in Section 2. Section 3 proposed some distance measures of SNLNs. In Section 4, the consistency-checking and consistency-improving issues of SNLPRs are discussed. Afterwards, there is an example and some analysis in Section 5. At last, some conclusions are drawn.

\section{Preliminaries}

In this section, some basic concepts and operations of linguistic term sets, SNLNs and LPRs, are reviewed.

A linguistic tem set is a collection of multiple linguistic values, like

$$
\bar{S}=\left\{s_{i} \mid i=-u, \ldots,-1,0,1, \ldots, u\right\},
$$

where $s_{i}$ is a possible linguistic value, and the negation operator is neg $\left(s_{i}\right)=s_{-i}$. Furthermore, if and only if $i>j$, then $s_{i}>s_{j}$ [66].

Note that the linguistic term set above is discrete. In some cases, the aggregated results may be used, which are not contained in this set. Hence, $\mathrm{Xu}$ [66] further defined a continuous term set, like

$$
S=\left\{s_{i} \mid i \in[-g, g]\right\}(g>u)
$$

to extend the old one.

The following are some operations of two linguistic terms $s_{i}, s_{j} \in S$.

$$
\begin{gathered}
s_{i} \oplus_{X u} s_{j}=s_{i+j} \\
s_{i} \oplus_{X u} s_{j}=s_{j} \oplus_{X u} s_{i} \\
\lambda s_{i}=s_{\lambda i}, 0 \leq \lambda \leq 1
\end{gathered}
$$

Definition 1. In Reference [67], let $S=\left\{s_{i} \mid i \in[-g, g]\right\}$ be a linguistic term set. The subscript of any element $s_{i}$ can be obtained by the function $N\left(s_{i}\right)=i$. The inverse function is $N^{-1}(i)=s_{i}$.

Definition 2. In Reference [68], suppose a crisp number $\vartheta_{i} \in[0,1]$. If there is a mapping from $s_{i}$ to $\vartheta_{i}$, then the linguistic scale function $f^{*}$ is denoted as $f^{*}: s_{i} \rightarrow \vartheta_{i}(i=-g,-g+1, \cdots, g-1, g)$, where $0 \leq \vartheta_{-g}<\vartheta_{-g+1}<\cdots<\vartheta_{g-1}<\vartheta_{g}$. And $f^{*-1}$ is the inverse function of $f^{*}$.

The linguistic scale function $f^{*}\left(s_{i}\right)=\frac{1}{2}+\frac{i}{2 g}(i \in[-g, g])$ is used in this paper.

Definition 3. In References [69,70], let $S=\left\{s_{i} \mid i \in[-g, g]\right\}$ be a linguistic term set. $\eta=\left\langle h_{\eta},\left(T_{\eta}, I_{\eta}, F_{\eta}\right)>\right.$ is a SNLN, where $h_{\eta}(x) \in S$, the truth-membership degree $T_{\eta}(x) \in[0,1]$, indeterminacy-membership degree $I_{\eta}(x) \in[0,1]$, and falsity-membership degree $F_{\eta}(x) \in[0,1]$, and $0 \leq T_{\eta}(x)+I_{\eta}(x)+F_{\eta}(x) \leq 3$.

Definition 4. In Reference [71], $A=<h_{A},\left(T_{A}, I_{A}, F_{A}\right)>$ and $B=<h_{B},\left(T_{B}, I_{B}, F_{B}\right)>$ are two arbitrary SNLNs, and their operations can be defined as follows:

(1) If $S(a)>S(b)$, then $a>b$;

(2) If $S(a)=S(b)$ and $A(a)>A(b)$, then $a>b$; 
(3) If $S(a)=S(b), A(a)=A(b)$ and $C(a)>C(b)$, then $a>b$;

(4) If $S(a)=S(b), A(a)=A(b)$ and $C(a)=C(b)$, then $a=b$.

Definition 5. In Reference [71], assume $a_{i}=\left(s_{\theta\left(a_{i}\right)},<T_{i}, I_{i}, F_{i}>\right)(i=1,2, \ldots, n)$ are a sequence of SNLNs. Then the simplified neutrosophic linguistic arithmetic mean (SNLAM) operator is

$$
\begin{aligned}
& \operatorname{SNLAM}\left(a_{1}, a_{2}, \ldots, a_{n}\right)=\bigoplus_{i=1}^{n} \frac{1}{n} a_{i} \\
& =\left\langle f^{*-1}\left(\frac{1}{n} \sum_{i=1}^{n}\left(f^{*}\left(s_{\theta\left(a_{i}\right)}\right)\right)\right),\left(\frac{\sum_{i=1}^{n}\left(f^{*}\left(s_{\theta\left(a_{i}\right)}\right) T_{i}\right)}{\sum_{i=1}^{n} f^{*}\left(s_{\theta\left(a_{i}\right)}\right)}, \frac{\sum_{i=1}^{n}\left(f^{*}\left(s_{\theta\left(a_{i}\right)}\right) I_{i}\right)}{\sum_{i=1}^{n} f^{*}\left(s_{\theta\left(a_{i}\right)}\right)}, \frac{\sum_{i=1}^{n}\left(f^{*}\left(s_{\theta\left(a_{i}\right)}\right) F_{i}\right)}{\sum_{i=1}^{n} f^{*}\left(s_{\theta\left(a_{i}\right)}\right)}\right)\right\rangle
\end{aligned}
$$

Definition 6. In Reference [71], suppose $a_{i}=\left(s_{\theta\left(a_{i}\right)},<T_{i}, I_{i}, F_{i}>\right)(i=1,2, \ldots, n)$ are a sequence of SNLNs. Then the simplified neutrosophic linguistic geometric mean (SNLGM) operator is

$$
\operatorname{SNLGM}\left(a_{1}, a_{2}, \ldots, a_{n}\right)=\stackrel{\otimes}{i=1}_{i}^{n} a_{i}^{\frac{1}{n}}=\left\langle f^{*-1}\left(\prod_{i=1}^{n}\left(f^{*}\left(s_{\theta\left(a_{i}\right)}\right)\right)^{\frac{1}{n}}\right),\left(\prod_{i=1}^{n} T_{i}^{\frac{1}{n}}, 1-\prod_{i=1}^{n}\left(1-I_{i}\right)^{\frac{1}{n}}, 1-\prod_{i=1}^{n}\left(1-F_{i}\right)^{\frac{1}{n}}\right)\right\rangle .
$$

Definition 7. In Reference [71], let $a=<h_{a},\left(T_{a}, I_{a}, F_{a}\right)>$ be a SNLN. Then the score function is $S F(a)=\frac{1}{3} f^{*}\left(h_{a}\right)\left(T_{a}+1-I_{a}+1-F_{a}\right)$, the accuracy function is $A F(a)=f^{*}\left(h_{a}\right)\left(T_{a}-F_{a}\right)$, and the certainty function is $\operatorname{CF}(a)=f^{*}\left(h_{a}\right) T_{a}$.

Definition 8. In Reference [71], for two SNLNs $a=<h_{a},\left(T_{a}, I_{a}, F_{a}\right)>$ and $b=<h_{b},\left(T_{b}, I_{b}, F_{b}\right)>$, the comparison method is:

(1) If $S(a)>S(b)$, then $a>b$;

(2) If $S(a)=S(b)$ and $A(a)>A(b)$, then $a>b$;

(3) If $S(a)=S(b), A(a)=A(b)$ and $C(a)>C(b)$, then $a>b$;

(4) If $S(a)=S(b), A(a)=A(b)$ and $C(a)=C(b)$, then $a=b$.

Definition 9. In Reference [72], let $X=\left\{x_{1}, x_{2}, \ldots, x_{n}\right\}$ be a collection of $n$ alternatives and $B=\left(b_{i j}\right)_{n \times n} \subset$ $X \times X$ be a judgment matrix. If for all $i, j=1,2, \ldots, n$, there are

$$
b_{i j} \oplus b_{j i}=s_{0} \text { and } b_{i i}=s_{0},
$$

then $B=\left(b_{i j}\right)_{n \times n}$ is a LPR, where $b_{i j}$ is the preference degree of the alternative $x_{i}$ over $x_{j}$. In particular, if $b_{i j}<s_{0}, x_{i}$ is non-preferred to $x_{j} ; x_{i}$ is preferred to $x_{j}$ if $b_{i j}>s_{0}$; if not, $x_{j}$ is equivalent to $x_{i}$.

Definition 10. In References [72,73], if $B=\left(b_{i j}\right)_{n \times n} \subset X \times X$ is a LPR, and

$$
b_{i j}=b_{i k} \oplus b_{k j},(i, k, j=1,2, \ldots, n)
$$

then $B$ is a perfectly consistent $L P R$.

\section{Distance Measures of SNLNs}

Ye [70] defined the distance measure between two SNLNs, but this method has some drawbacks. Thus, some distance measures of SNLNs are redefined in this section.

Distance measure is a universal and effective way to calculate the difference between two elements. There are several common distance measures, such as Hamming distance, Euclidean distance, and Hausdorff distance. 
Definition 11. In Reference [70], suppose $\alpha=\left(T_{\alpha}, I_{\alpha}, F_{\alpha}\right)$ and $\beta=\left(T_{\beta}, I_{\beta}, F_{\beta}\right)$ are two optional SNLNs, and the subscript function is $N\left(s_{i}\right)=i$. $\lambda \geq 0$. The distance between $\alpha$ and $\beta$ can be defined as below:

$$
d_{Y}(\alpha, \beta)=\left(\left|T_{\alpha} \cdot N\left(h_{\alpha}\right)-T_{\beta} \cdot N\left(h_{\beta}\right)\right|_{\lambda}+\left|I_{\alpha} \cdot N\left(h_{\alpha}\right)-I_{\beta} \cdot N\left(h_{\beta}\right)\right|_{\lambda}+\left|F_{\alpha} \cdot N\left(h_{\alpha}\right)-F_{\beta} \cdot N\left(h_{\beta}\right)\right|_{\lambda}\right)^{\frac{1}{\lambda}} .
$$

Specially, when $\lambda=1$, Equation (8) can be reduced to Hamming distance; when $\lambda=2$, Equation (8) can be reduced to Euclidean distance.

The limitations of this definition are noticeable. Firstly, the calculation depends on linguistic subscripts directly, and different semantics cannot be distinguished. Secondly, this distance does not satisfy $0 \leq d_{Y}(\alpha, \beta) \leq 1$ and the property of triangle inequality. Thirdly, the truth-membership, indeterminacy-membership, and false-membership are put on an equal footing in the calculation process. This is intuitively irrational.

To overcome these shortcomings, the following distance measures between two SNLNs are defined.

Definition 12. For two arbitrary SNLNs $a=<h_{a},\left(T_{a}, I_{a}, F_{a}\right)>$ and $b=<h_{b},\left(T_{b}, I_{b}, F_{b}\right)>$, assume the linguistic term set is $S=\left\{s_{i} \mid i \in[-g, g]\right\}$ and the subscript function is $N\left(s_{i}\right)=i$. Then the Hamming distance $d_{H}(a, b)$, Euclidean distance $d_{E}(a, b)$, and Hausdorff distance $d_{H a}(a, b)$ can be defined as follows:

$$
\begin{gathered}
d_{H}(a, b)=\frac{1}{6 g}\left[N\left(\left|T_{a} \cdot h_{a}-T_{b} \cdot h_{b}\right|\right)+N\left(\left|\left(1-I_{a}\right) \cdot h_{a}-\left(1-I_{b}\right) \cdot h_{b}\right|\right)+N\left(\left|\left(1-F_{a}\right) \cdot h_{a}-\left(1-F_{b}\right) \cdot h_{b}\right|\right)\right], \\
d_{E}(a, b)=\sqrt{\frac{1}{12 g^{2}}\left[N\left(\left|T_{a} \cdot h_{a}-T_{b} \cdot h_{b}\right|\right)^{2}+N\left(\left|\left(1-I_{a}\right) \cdot h_{a}-\left(1-I_{b}\right) \cdot h_{b}\right|\right)^{2}+N\left(\left|\left(1-F_{a}\right) \cdot h_{a}-\left(1-F_{b}\right) \cdot h_{b}\right|\right)^{2}\right]}, \\
d_{H a}(a, b)=\frac{1}{2 g} \max \left\{N\left(\left|T_{a} \cdot h_{a}-T_{b} \cdot h_{b}\right|\right),\left(\left|\left(1-I_{a}\right) \cdot h_{a}-\left(1-I_{b}\right) \cdot h_{b}\right|\right), N\left(\left|\left(1-F_{a}\right) \cdot h_{a}-\left(1-F_{b}\right) \cdot h_{b}\right|\right)\right\} .
\end{gathered}
$$

Property 1. Assume $\Omega$ is the set of all SNLNs, $a=<h_{a},\left(T_{a}, I_{a}, F_{a}\right)>, b=<h_{b},\left(T_{b}, I_{b}, F_{b}\right)>$ and $c=<h_{\mathcal{c}},\left(T_{\mathcal{C}}, I_{\mathcal{C}}, F_{\mathcal{c}}\right)>, S=\left\{s_{i} \mid i \in[-g, g]\right\}$, and then the following properties are satisfied:

(1) $0 \leq d_{H}(a, b) \leq 1,0 \leq d_{E}(a, b) \leq 1$, and $0 \leq d_{H a}(a, b) \leq 1$, for $\forall a, b \in \Omega$;

(2) $\quad d_{H}(a, b)=d_{H}(b, a), d_{E}(a, b)=d_{E}(b, a)$, and $d_{H a}(a, b)=d_{H a}(b, a)$, for $\forall a, b \in \Omega$;

(3) If $a=b$, then $d_{H}(a, b)=0, d_{E}(a, b)=0$, and $d_{H a}(a, b)=0$, for $\forall a, b \in \Omega$;

(4) If $s_{0} \leq h_{a} \leq h_{b} \leq h_{c}, T_{a} \leq T_{b} \leq T_{c}, I_{a} \geq I_{b} \geq I_{c}$ and $F_{a} \geq F_{b} \geq F_{c}$, then $d_{H}(a, b) \leq d_{H}(a, c)$, $d_{H}(b, c) \leq d_{H}(a, c), d_{E}(a, b) \leq d_{E}(a, c), d_{E}(b, c) \leq d_{E}(a, c), d_{H a}(a, b) \leq d_{H a}(a, c)$ and $d_{H a}(b, c) \leq$ $d_{H a}(a, c)$.

\section{Proof 1.}

(1) Because $i \in[-g, g]$ and $0 \leq T_{a}, T_{b} \leq 1, N\left(\left|T_{a} \cdot h_{a}-T_{b} \cdot h_{b}\right|\right) \in[0,2 g]$; Similarly, $0 \leq\left(1-I_{a}\right) \leq 1,0 \leq\left(1-I_{b}\right) \leq 1 \Rightarrow N\left(\left|\left(1-I_{a}\right) \cdot h_{a}-\left(1-I_{b}\right) \cdot h_{b}\right|\right) \in[0,2 g]$, and $0 \leq\left(1-F_{a}\right) \leq 1,0 \leq\left(1-F_{b}\right) \leq 1 \Rightarrow N\left(\left|\left(1-F_{a}\right) \cdot h_{a}-\left(1-F_{b}\right) \cdot h_{b}\right|\right) \in[0,2 g]$; thus $0 \leq\left[N\left(\left|T_{a} \cdot h_{a}-T_{b} \cdot h_{b}\right|\right)+N\left(\left|\left(1-I_{a}\right) \cdot h_{a}-\left(1-I_{b}\right) \cdot h_{b}\right|\right)+N\left(\left|\left(1-F_{a}\right) \cdot h_{a}-\left(1-F_{b}\right) \cdot h_{b}\right|\right)\right]$ $\leq 6 g \Rightarrow 0 \leq d_{H}(a, b) \leq 1$. Likewise, $0 \leq d_{E}(a, b) \leq 1$ and $0 \leq d_{H a}(a, b) \leq 1$.

(2) $\quad N\left(\left|T_{a} \cdot h_{a}-T_{b} \cdot h_{b}\right|\right)=N\left(\left|T_{b} \cdot h_{b}-T_{a} \cdot h_{a}\right|\right), N\left(\left|\left(1-I_{a}\right) \cdot h_{a}-\left(1-I_{b}\right) \cdot h_{b}\right|\right)=N\left(\mid\left(1-I_{b}\right) \cdot h_{b}-\right.$ $\left.\left(1-I_{a}\right) \cdot h_{a} \mid\right)$, and $N\left(\left|\left(1-F_{a}\right) \cdot h_{a}-\left(1-F_{b}\right) \cdot h_{b}\right|\right)=N\left(\left|\left(1-F_{b}\right) \cdot h_{b}-\left(1-F_{a}\right) \cdot h_{a}\right|\right)$, therefore $d_{H}(a, b)=d_{H}(b, a)$. Likewise, $d_{E}(a, b)=d_{E}(b, a)$ and $d_{H a}(a, b)=d_{H a}(b, a)$.

(3) $\quad a=b \Rightarrow N\left(\left|T_{a} \cdot h_{a}-T_{b} \cdot h_{b}\right|\right)=0, N\left(\left|\left(1-I_{a}\right) \cdot h_{a}-\left(1-I_{b}\right) \cdot h_{b}\right|\right)=0$ and $N\left(\mid\left(1-F_{a}\right) \cdot h_{a}-(1-\right.$ $\left.\left.F_{b}\right) \cdot h_{b} \mid\right)=0$, therefore $d_{H}(a, b)=0$. Similarly, $d_{E}(a, b)=0$ and $d_{H a}(a, b)=0$.

(4) Because $s_{0} \leq s a_{i j} \leq s b_{i j} \leq s c_{i j}, T_{i j}^{a} \leq T_{i j}^{b} \leq T_{i j}^{c}$, and $N\left(s_{i}\right)=i$ is a monotone increasing function, $T_{i j}^{a} \cdot\left(s a_{i j}\right) \leq T_{i j}^{b} \cdot\left(s b_{i j}\right) \leq T_{i j}^{c} \cdot\left(s c_{i j}\right) \Rightarrow\left|T_{i j}^{a} \cdot\left(s a_{i j}\right)-T_{i j}^{b} \cdot\left(s b_{i j}\right)\right| \leq\left|T_{i j}^{a} \cdot\left(s a_{i j}\right)-T_{i j}^{c} \cdot\left(s c_{i j}\right)\right|$ and $N\left(\left|T_{i j}^{a} \cdot\left(s a_{i j}\right)-T_{i j}^{b} \cdot\left(s b_{i j}\right)\right|\right) \leq N\left(\left|T_{i j}^{a} \cdot\left(s a_{i j}\right)-T_{i j}^{c} \cdot\left(s c_{i j}\right)\right|\right)$; Likewise, $N\left(\mid\left(1-I_{i j}^{a}\right) \cdot\left(s a_{i j}\right)-(1-\right.$ $\left.\left.I_{i j}^{b}\right) \cdot\left(s b_{i j}\right) \mid\right) \leq N\left(\left|\left(1-I_{i j}^{a}\right) \cdot\left(s a_{i j}\right)-\left(1-I_{i j}^{c}\right) \cdot\left(s c_{i j}\right)\right|\right)$ and $N\left(\left|\left(1-F_{i j}^{a}\right) \cdot\left(s a_{i j}\right)-\left(1-F_{i j}^{b}\right) \cdot\left(s b_{i j}\right)\right|\right) \leq$ $N\left(\left|\left(1-F_{i j}^{a}\right) \cdot\left(s a_{i j}\right)-\left(1-F_{i j}^{c}\right) \cdot\left(s c_{i j}\right)\right|\right)$, so $d_{H}(a, b) \leq d_{H}(a, c)$. Similarly, $d_{H}(b, c) \leq d_{H}(a, c)$, $d_{E}(a, b) \leq d_{E}(a, c), d_{E}(b, c) \leq d_{E}(a, c), d_{H a}(a, b) \leq d_{H a}(a, c)$ and $d_{H a}(b, c) \leq d_{H a}(a, c)$. 
Then, the proof is completed.

Example 1. $a=<s_{1},(0.2,0.3,0.6)>$ and $b=<s_{2},(0.5,0.1,0.4)>$ are two SNLNs, and $g=4$. Then $d_{H}(a, b) \approx 0.1125, d_{E}(a, b) \approx 0.1139$ and $d_{H a}(a, b) \approx 0.1375$.

\section{Decision-Making Method Based on SNLPRs}

In this section, the concept of SNLPRs is presented. A decision-making method is proposed after discussing the checking and improving of consistency.

\subsection{The Concept of SNLPRS}

Definition 13. Given a group of $n$ alternatives $X=\left\{x_{1}, x_{2}, \ldots, x_{n}\right\}$ and a matrix $K=\left(k_{i j}\right)_{n \times n} \subset X \times X$. If all the elements are presented with SNLNs, $k_{i j}=\left(s_{i j},<T_{i j}, I_{i j}, F_{i j}>\right)$, and satisfy these conditions in the following:

$$
\begin{gathered}
s_{i j} \oplus_{X u} s_{j i}=s_{0} \\
T_{i j}=T_{j i}, I_{i j}=I_{j i}, F_{i j}=F_{j i} \\
\left(s_{i i},<T_{i i}, I_{i i}, F_{i i}>\right)=\left(s_{0},<1,0,0>\right),(i, j=1,2, \ldots, n),
\end{gathered}
$$

then the matrix $K$ on $X$ can be regarded as a SNLPR, where $s_{i j}$ is the degree of $x_{i}$ preferred to $x_{j}$, and $T_{i j}, I_{i j}$ and $F_{i j}$ represent the truth-membership degree, the indeterminacy-membership degree, and the falsity-membership degree of $s_{i j}$, respectively.

Specifically, when $T_{i j}=1$ and $I_{i j}=F_{i j}=0$ for all $i, j=1,2, \ldots, n$, then the SNLPR is reduced to a LPR. Compared to LPRs, SNLPRs contain not only the linguistic values, but also the degrees of accuracy, hesitation, and mistake. The discrete linguistic term set can be extended to be a continuous one and DMs can express their qualitative preference information more flexibly.

From Definition 13, it can be seen that $k_{i j}=\left(s_{i j},<T_{i j}, I_{i j}, F_{i j}>\right)$ is the preferred value of the scheme $x_{i}$ to $x_{j}$, and it could be the same as $k_{i j}=\left(<s_{i j}, T_{i j}>,<s_{i j}, I_{i j}>,<s_{i j}, F_{i j}>\right)$, where $<s_{i j}, T_{i j}>$ shows $x_{i}$ is $s_{i j}$ to $x_{j}$ with the true possibility $T_{i j}$; $<s_{i j}, I_{i j}>$ shows $x_{i}$ is $s_{i j}$ to $x_{j}$ with the hesitant possibility $I_{i j} ;<s_{i j}, F_{i j}>$ shows $x_{i}$ is $s_{i j}$ to $x_{j}$ with the false possibility $F_{i j}$.

As well as LPR, the preference degree of $x_{j}$ to $x_{i}$ can be denoted as $\left\langle s_{-i j}, T_{i j}\right\rangle,\left\langle s_{-i j}, I_{i j}\right\rangle$ and $\left\langle s_{-i j}, F_{i j}>\right.$, individually. That is to say, $\left.k_{j i}=\left(<s_{-i j}, T_{i j}\right\rangle,\left\langle s_{-i j}, I_{i j}\right\rangle,\left\langle s_{-i j}, F_{i j}\right\rangle\right)$ or $k_{j i}=\left(s_{-i j},<T_{i j}, I_{i j}, F_{i j}>\right)$.

Example 2. There are three alternatives $X=\left\{x_{1}, x_{2}, x_{3}\right\}$, and the linguistic term set $S=\left\{s_{i} \mid i \in[-4,4]\right\}$ is used, where $s=\left\{s_{-4}=\right.$ tremendously poorer, $s_{-3}=$ much poorer, $s_{-2}=$ poorer,$s_{-1}=$ a little poorer, $s_{0}=$ fair, $s_{1}=$ a little better,$s_{2}=$ better,$s_{3}=$ much better, $s_{4}=$ tremendously better $\}$. If a decision maker believes the degree of $x_{1}$ preferred to $x_{2}$ is $s_{2}$, but he is not sure that he is absolutely right. According to his professional knowledge and experience in the past, he deems that he is correct with a probability of $40 \%$, but the probability of error is $50 \%$, and the uncertainty is $10 \%$. In that case, his preference can be described using a SNLN, that is, $\left(s_{2},<0.4,0.1,0.5>\right)$. In this way, a SNLPR can be obtained as all the alternatives above are compared with each other in a proper sequence. An example is given as follows:

$$
K_{1}=\left[\begin{array}{ccc}
\left(s_{0},<1,0,0>\right) & \left(s_{-1},<0.4,0.2,0.1>\right) & \left(s_{2},<0.3,0.1,0.2>\right) \\
\left(s_{1},<0.4,0.2,0.1>\right) & \left(s_{0},<1,0,0>\right) & \left(s_{-3},<0.2,0.5,0.3>\right) \\
\left(s_{-2},<0.3,0.1,0.2>\right) & \left(s_{3},<0.2,0.5,0.3>\right) & \left(s_{0},<1,0,0>\right)
\end{array}\right] .
$$

Definition 14. Let $X=\left\{x_{1}, x_{2}, \ldots, x_{n}\right\}$ be a cluster of $n$ alternatives and a SNLPR be $K=\left(k_{i j}\right)_{n \times n} \subset X \times X$, where $k_{i j}=\left(s_{i j},<T_{i j}, I_{i j}, F_{i j}>\right)$. Then the matrix $T=\left(<s_{i j}, T_{i j}>\right)_{n \times n} \subset X \times X$ is regarded as the true 
linguistic judgment matrix of $\left.K, I=\left(<s_{i j}, I_{i j}\right\rangle\right)_{n \times n} \subset X \times X$ is the hesitant linguistic judgment matrix of $K$, and $F=\left(<s_{i j}, F_{i j}>\right)_{n \times n} \subset X \times X$ is the false linguistic judgment matrix of $K$, respectively.

From Definition 14, it can be known that for an arbitrary SNLPR, it is easy to derive its corresponding true linguistic judgment matrix, hesitant linguistic judgment matrix, and false linguistic judgment matrix. Furthermore, these linguistic judgment matrices are all defined based on the continuous linguistic terms.

Example 3. Suppose a SNLPR is the same as in Example 2. Then, according to Definition 14, its corresponding true linguistic judgment matrix, hesitant linguistic judgment matrix, and false linguistic judgment matrix are $T_{1}=\left[\begin{array}{ccc}\left(<s_{0}, 1>\right) & \left(<s_{-1}, 0.4>\right) & \left(<s_{2}, 0.3>\right) \\ \left(<s_{1}, 0.4>\right) & \left(<s_{0}, 1>\right) & \left(<s_{-3}, 0.2>\right) \\ \left(<s_{-2}, 0.3>\right) & \left(<s_{3}, 0.2>\right) & \left(<s_{0}, 1>\right)\end{array}\right]$, $I_{1}=\quad\left[\begin{array}{ccc}\left(<s_{0}, 0>\right) & \left(<s_{-1}, 0.2>\right) & \left(<s_{2}, 0.1>\right) \\ \left(<s_{1}, 0.2>\right) & \left(<s_{0}, 0>\right) & \left(<s_{-3}, 0.5>\right) \\ \left(<s_{-2}, 0.1>\right) & \left(<s_{3}, 0.5>\right) & \left(<s_{0}, 0>\right)\end{array}\right]$ and $F_{1} \quad=$
$\left[\begin{array}{ccc}\left(<s_{0}, 0>\right) & \left(<s_{-1}, 0.1>\right) & \left(<s_{2}, 0.2>\right) \\ \left(<s_{1}, 0.1>\right) & \left(<s_{0}, 0>\right) & \left(<s_{-3}, 0.3>\right) \\ \left(<s_{-2}, 0.2>\right) & \left(<s_{3}, 0.3>\right) & \left(<s_{0}, 0>\right)\end{array}\right]$.

\subsection{Consistency Checking of SNLPRs}

The deviation between two SNLPRs is calculated in this subsection, and then a distance-based consistency index is presented as well.

Definition 15. Assume there are several alternatives $X=\left\{x_{1}, x_{2}, \ldots, x_{n}\right\}$. For an arbitrary SLNLPR, if for all $i, j, k=1,2, \ldots, n$, there is $\left(T_{i k} \cdot s_{i k}\right) \oplus\left(T_{k j} \cdot s_{k j}\right)=\left(T_{i j} \cdot s_{i j}\right)$, then it can be regarded that $T=\left(T_{i j} \cdot s_{i j}\right)_{n \times n} \subset$ $X \times X$ is consistent; if for all $i, j, k=1,2, \ldots, n,\left(\left(1-I_{i k}\right) \cdot s_{i k}\right) \oplus\left(\left(1-I_{k j}\right) \cdot s_{k j}\right)=\left(\left(1-I_{i j}\right) \cdot s_{i j}\right), I=$ $\left(I_{i j} \cdot s_{i j}\right)_{n \times n} \subset X \times X$ is consistent; Similarly, if for all $i, j, k=1,2, \ldots, n,\left(\left(1-F_{i k}\right) \cdot s_{i k}\right) \oplus\left(\left(1-F_{k j}\right) \cdot s_{k j}\right)=$ $\left.\left(\left(1-F_{i j}\right) \cdot s_{i j}\right), F=\left(<F_{i j} \cdot s_{i j}\right\rangle\right)_{n \times n} \subset X \times X$ is consistent.

Definition 16. Let $K=\left(k_{i j}\right)_{n \times n}=\left(s_{i j},<T_{i j}, I_{i j}, F_{i j}>\right)_{n \times n} \subset X \times X$ be a SNLPR. If the following equations are true for all $i, j, k=1,2, \ldots, n$ :

$$
\begin{gathered}
\left(T_{i k} \cdot s_{i k}\right) \oplus\left(T_{k j} \cdot s_{k j}\right)=\left(T_{i j} \cdot s_{i j}\right) \\
\left(\left(1-I_{i k}\right) \cdot s_{i k}\right) \oplus\left(\left(1-I_{k j}\right) \cdot s_{k j}\right)=\left(\left(1-I_{i j}\right) \cdot s_{i j}\right) \\
\left(\left(1-F_{i k}\right) \cdot s_{i k}\right) \oplus\left(\left(1-F_{k j}\right) \cdot s_{k j}\right)=\left(\left(1-F_{i j}\right) \cdot s_{i j}\right),
\end{gathered}
$$

then $K$ is regarded as a consistent SNLPR.

Example 4. Suppose a SNLPR is the same one as Example 2. Because $\left(T_{12} \cdot s_{12}\right) \oplus\left(T_{23} \cdot s_{23}\right)=0.4 s_{-1} \oplus$ $0.2 s_{-3}=s_{-1} \neq T_{13} \cdot s_{13}$, based on Definition $16, K_{1}$ is not considered a consistent SNLPR.

Theorem 1. Given some alternatives $X=\left\{x_{1}, x_{2}, \ldots, x_{n}\right\}$, and the related SNLPR is $K=\left(k_{i j}\right)_{n \times n}=$ $\left(s_{i j},<T_{i j}, I_{i j}, F_{i j}>\right)_{n \times n} \subset X \times X$. If $T=\left(T_{i j} \cdot s_{i j}\right)_{n \times n} \subset X \times X, I=\left(I_{i j} \cdot s_{i j}\right)_{n \times n} \subset X \times X$ and $F=$ $\left(F_{i j} \cdot s_{i j}\right)_{n \times n} \subset X \times X$ all have perfect consistency, then the SNLPR $K$ is consistent, too. On the contrary, when a SNLPR K has complete consistency, then $T=\left(T_{i j} \cdot s_{i j}\right)_{n \times n} \subset X \times X, I=\left(I_{i j} \cdot s_{i j}\right)_{n \times n} \subset X \times X$ and $F=\left(F_{i j} \cdot s_{i j}\right)_{n \times n} \subset X \times X$ are all absolutely consistent. 


\section{Proof 2.}

(1) Because $T=\left(T_{i j} \cdot s_{i j}\right)_{n \times n} \subset X \times X$ is consistent, for all $i, j, k=1,2, \ldots, n$, there is $\left(T_{i k} \cdot s_{i k}\right) \oplus$ $\left(T_{k j} \cdot s_{k j}\right)=\left(T_{i j} \cdot s_{i j}\right)$ based on Definition 15. In the same way, $\left(\left(1-I_{i k}\right) \cdot s_{i k}\right) \oplus\left(\left(1-I_{k j}\right) \cdot s_{k j}\right)=$ $\left(\left(1-I_{i j}\right) \cdot s_{i j}\right)$ and $\left(\left(1-F_{i k}\right) \cdot s_{i k}\right) \oplus\left(\left(1-F_{k j}\right) \cdot s_{k j}\right)=\left(\left(1-F_{i j}\right) \cdot s_{i j}\right)$, as $I=\left(I_{i j} \cdot s_{i j}\right)_{n \times n} \subset X \times X$ and $F=\left(F_{i j} \cdot s_{i j}\right)_{n \times n} \subset X \times X$ are consistent. That is to say, $\left(T_{i k} \cdot s_{i k}\right) \oplus\left(T_{k j} \cdot s_{k j}\right)=\left(T_{i j} \cdot s_{i j}\right)$, $\left(\left(1-I_{i k}\right) \cdot s_{i k}\right) \oplus\left(\left(1-I_{k j}\right) \cdot s_{k j}\right)=\left(\left(1-I_{i j}\right) \cdot s_{i j}\right)$ and $\left(\left(1-F_{i k}\right) \cdot s_{i k}\right) \oplus\left(\left(1-F_{k j}\right) \cdot s_{k j}\right)=((1-$ $\left.\left.F_{i j}\right) \cdot s_{i j}\right)$ for all $i, j, k=1,2, \ldots, n$. On the basis of Definition 16 , it can be seen that $K$ is consistent.

(2) Since $K$ has complete consistency, then these equations hold based on Definition 16: $\left(T_{i k} \cdot s_{i k}\right) \oplus$ $\left(T_{k j} \cdot s_{k j}\right)=\left(T_{i j} \cdot s_{i j}\right),\left(\left(1-I_{i k}\right) \cdot s_{i k}\right) \oplus\left(\left(1-I_{k j}\right) \cdot s_{k j}\right)=\left(\left(1-I_{i j}\right) \cdot s_{i j}\right)$ and $\left(\left(1-F_{i k}\right) \cdot s_{i k}\right) \oplus$ $\left(\left(1-F_{k j}\right) \cdot s_{k j}\right)=\left(\left(1-F_{i j}\right) \cdot s_{i j}\right)$. In the light of Definition 15, $T=\left(T_{i j} \cdot s_{i j}\right)_{n \times n} \subset X \times X$, $I=\left(I_{i j} \cdot s_{i j}\right)_{n \times n} \subset X \times X$ and $F=\left(F_{i j} \cdot s_{i j}\right)_{n \times n} \subset X \times X$ are all consistent as well.

The proof is done now.

Theorem 2. Given an arbitrary SNLPR $K=\left(k_{i j}\right)_{n \times n}=\left(s_{i j},<T_{i j}, I_{i j}, F_{i j}>\right)_{n \times n} \subset X \times X, i, j, k=$ $1,2, \ldots n$, if

$$
\begin{gathered}
T_{i j}^{*} \cdot s_{i j}^{*}=\frac{1}{n}\left\{\oplus_{k=1}^{n}\left[\left(T_{i k} \cdot s_{i k}\right) \oplus\left(T_{k j} \cdot s_{k j}\right)\right]\right\} \\
\left(1-I_{i j}^{*}\right) \cdot s_{i j}^{*}=\frac{1}{n}\left\{\oplus_{k=1}^{n}\left[\left(\left(1-I_{i k}\right) \cdot s_{i k}\right) \oplus\left(\left(1-I_{k j}\right) \cdot s_{k j}\right)\right]\right\} \\
\left(1-F_{i j}^{*}\right) \cdot s_{i j}^{*}=\frac{1}{n}\left\{\oplus_{k=1}^{n}\left[\left(\left(1-F_{i k}\right) \cdot s_{i k}\right) \oplus\left(\left(1-F_{k j}\right) \cdot s_{k j}\right)\right]\right\},
\end{gathered}
$$

then a consistent SNLPR $K^{*}=\left(k_{i j}^{*}\right)_{n \times n}=\left(s_{i j}^{*},<T_{i j}^{*}, I_{i j}^{*}, F_{i j}^{*}>\right)_{n \times n}$ is obtained.

Proof 3. Since $\left(T_{i k}^{*} \cdot s_{i k}^{*}\right) \oplus\left(T_{k j}^{*} \cdot s_{k j}^{*}\right)=\frac{1}{n}\left\{\oplus_{e=1}^{n}\left[\left(T_{i e} \cdot s_{i e}\right) \oplus\left(T_{e k} \cdot s_{e k}\right)\right]\right\} \oplus \frac{1}{n}\left\{\oplus_{e=1}^{n}\left[\left(T_{k e} \cdot s_{k e}\right) \oplus\left(T_{e j} \cdot s_{e j}\right)\right]\right\}$ $=\frac{1}{n}\left\{\oplus_{e=1}^{n}\left[\left(T_{i e} \cdot s_{i e}\right) \oplus\left(T_{e k} \cdot s_{e k}\right) \oplus\left(T_{k e} \cdot s_{k e}\right) \oplus\left(T_{e j} \cdot s_{e j}\right)\right]\right\}=\frac{1}{n}\left\{\oplus_{e=1}^{n}\left[\left(T_{i e} \cdot s_{i e}\right) \oplus\left(T_{e j} \cdot s_{e j}\right) \oplus\left(T_{e k} \cdot s_{0}\right)\right]\right\}$ $=\frac{1}{n}\left\{\oplus_{e=1}^{n}\left[\left(T_{i e} \cdot s_{i e}\right) \oplus\left(T_{e j} \cdot s_{e j}\right)\right]\right\}=T_{i j}^{*} \cdot s_{i j}^{*}$; then $\left(T_{i k}^{*} \cdot s_{i k}^{*}\right) \oplus\left(T_{k j}^{*} \cdot s_{k j}^{*}\right)=T_{i j}^{*} \cdot s_{i j}^{*} ;$ Similarly, $\left(\left(1-I_{i k}^{*}\right) \cdot s_{i k}^{*}\right) \oplus$ $\left(\left(1-I_{k j}^{*}\right) \cdot s_{k j}^{*}\right)=\frac{1}{n}\left\{\oplus_{e=1}^{n}\left[\left(\left(1-I_{i e}\right) \cdot s_{i e}\right) \oplus\left(\left(1-I_{e k}\right) \cdot s_{e k}\right)\right]\right\} \oplus \frac{1}{n}\left\{\oplus_{e=1}^{n}\left[\left(\left(1-I_{k e}\right) \cdot s_{k e}\right) \oplus\left(\left(1-I_{e j}\right) \cdot s_{e j}\right)\right]\right\}$ $=\frac{1}{n}\left\{\oplus_{e=1}^{n}\left[\left(\left(1-I_{i e}\right) \cdot s_{i e}\right) \oplus\left(\left(1-I_{e k}\right) \cdot s_{e k}\right) \oplus\left(\left(1-I_{k e}\right) \cdot s_{k e}\right) \oplus\left(\left(1-I_{e j}\right) \cdot s_{e j}\right)\right]\right\}=\frac{1}{n}\left\{\oplus_{e=1}^{n}\left[\left(\left(1-I_{i e}\right) \cdot s_{i e}\right)\right.\right.$ $\left.\left.\oplus\left(\left(1-I_{e j}\right) \cdot s_{e j}\right) \oplus\left(\left(1-I_{e k}\right) \cdot s_{0}\right)\right]\right\}=\frac{1}{n}\left\{\oplus_{e=1}^{n}\left[\left(\left(1-I_{i e}\right) \cdot s_{i e}\right) \oplus\left(\left(1-I_{e j}\right) \cdot s_{e j}\right)\right]\right\}$ $=\left(1-I_{i j}^{*}\right) \cdot s_{i j}^{*}$ and $\left(\left(1-F_{i k}^{*}\right) \cdot s_{i k}^{*}\right) \oplus\left(\left(1-F_{k j}^{*}\right) \cdot s_{k j}^{*}\right)=\frac{1}{n}\left\{\oplus_{e=1}^{n}\left[\left(\left(1-F_{i e}\right) \cdot s_{i e}\right) \oplus\left(\left(1-F_{e k}\right) \cdot s_{e k}\right)\right]\right\} \oplus$ $\frac{1}{n}\left\{\oplus_{e=1}^{n}\left[\left(\left(1-F_{k e}\right) \cdot s_{k e}\right) \oplus\left(\left(1-F_{e j}\right) \cdot s_{e j}\right)\right]\right\}=\frac{1}{n}\left\{\oplus_{e=1}^{n}\left[\left(\left(1-F_{i e}\right) \cdot s_{i e}\right) \oplus\left(\left(1-F_{e k}\right) \cdot s_{e k}\right) \oplus\left(\left(1-F_{k e}\right) \cdot s_{k e}\right)\right.\right.$ $\left.\left.\oplus\left(\left(1-F_{e j}\right) \cdot s_{e j}\right)\right]\right\}=\frac{1}{n}\left\{\oplus_{e=1}^{n}\left[\left(\left(1-F_{i e}\right) \cdot s_{i e}\right) \oplus\left(\left(1-F_{e j}\right) \cdot s_{e j}\right) \oplus\left(\left(1-F_{e k}\right) \cdot s_{0}\right)\right]\right\}=\frac{1}{n}\left\{\oplus_{e=1}^{n}\left[\left(\left(1-F_{i e}\right) \cdot\right.\right.\right.$ $\left.\left.\left.s_{i e}\right) \oplus\left(\left(1-F_{e j}\right) \cdot s_{e j}\right)\right]\right\}=\left(1-F_{i j}^{*}\right) \cdot s_{i j}^{*}$

According to Equations (15)-(17), it can be seen that $K^{*}=\left(k_{i j}^{*}\right)_{n \times n}=\left(s_{i j}^{*},<T_{i j}^{*}, I_{i j}^{*}, F_{i j}^{*}>\right)_{n \times n}$ is a consistent SNLPR.

This is the end of Proof 3.

Note that there are only three equations above, but four variables $s_{i j}^{*}, T_{i j}^{*}, I_{i j}^{*}$ and $F_{i j}^{*}$ are contained. Thus, there may be many possible answers. In order to get a unique solution, the following method is used:

(1) In a general way, assume $s_{i j}^{*} \geq s_{0}$, and then $s_{0} \leq T_{i j}^{*} \cdot s_{i j}^{*} \leq s_{i j}^{*}, s_{o} \leq\left(1-I_{i j}^{*}\right) \cdot s_{i j}^{*} \leq s_{i j}^{*}$, and $s_{o} \leq\left(1-F_{i j}^{*}\right) \cdot s_{i j}^{*} \leq s_{i j}^{*}$; suppose $\max \left\{N\left(T_{i j}^{*} \cdot s_{i j}^{*}\right), N\left(\left(1-I_{i j}^{*}\right) \cdot s_{i j}^{*}\right), N\left(\left(1-F_{i j}^{*}\right) \cdot s_{i j}^{*}\right)\right\} \in[a-1, a]$, $a \leq N\left(s_{i j}^{*}\right)$, and then a unique SNLN $\left(s_{i j}^{*},<T_{i j}^{*}, I_{i j}^{*}, F_{i j}^{*}>\right)=\left(s_{a}, \frac{N\left(T_{i j}^{*} \cdot s_{i j}^{*}\right)}{a}, 1-\frac{N\left(\left(1-I_{i j}^{*}\right) \cdot s_{i j}^{*}\right)}{a}, 1-\frac{N\left(\left(1-F_{i j}^{*}\right) \cdot s_{i j}^{*}\right)}{a}\right)$ can be gained.

For instance, if $T_{i j}^{*} \cdot s_{i j}^{*}=0.3,\left(1-I_{i j}^{*}\right) \cdot s_{i j}^{*}=1.2$, and $\left(1-F_{i j}^{*}\right) \cdot s_{i j}^{*}=2.7$, there is $\max \left\{N\left(T_{i j}^{*}\right.\right.$. $\left.\left.s_{i j}^{*}\right), N\left(\left(1-I_{i j}^{*}\right) \cdot s_{i j}^{*}\right), N\left(\left(1-F_{i j}^{*}\right) \cdot s_{i j}^{*}\right)\right\}=\max (0.3,1.2,2.7)=2.7 \in[2,3]$, so $a=3$ and $\left(s_{i j}^{*},<T_{i j}^{*}, I_{i j}^{*}, F_{i j}^{*}>\right)$ $=\left(s_{3},<0.1,0.6,0.1>\right)$. 
(2) For $s_{i j}^{*} \leq s_{0}, s_{i j}^{*} \leq T_{i j}^{*} \cdot s_{i j}^{*} \leq s_{0}, s_{i j}^{*} \leq\left(1-I_{i j}^{*}\right) \cdot s_{i j}^{*} \leq s_{o}$ and $s_{i j}^{*} \leq\left(1-F_{i j}^{*}\right) \cdot s_{i j}^{*} \leq s_{0}$; if $\min \left\{N\left(T_{i j}^{*} \cdot\right.\right.$ $\left.\left.s_{i j}^{*}\right), N\left(\left(1-I_{i j}^{*}\right) \cdot s_{i j}^{*}\right), N\left(\left(1-F_{i j}^{*}\right) \cdot s_{i j}^{*}\right)\right\} \in[a, a+1], a \geq N\left(s_{i j}^{*}\right)$, then a solitary SNLN is $\left(s_{i j}^{*},<T_{i j}^{*}, I_{i j}^{*}, F_{i j}^{*}>\right)$ $=\left(s_{a}, \frac{N\left(T_{i j}^{*} \cdot s_{i j}^{*}\right)}{a}, 1-\frac{N\left(\left(1-I_{i j}^{*}\right) \cdot F_{i j}^{*}\right)}{a}, 1-\frac{N\left(\left(1-F_{i j}^{*}\right) \cdot s_{i j}^{*}\right)}{a}\right)$.

For example, $T_{i j}^{*} \cdot s_{i j}^{*}=-0.3,\left(1-I_{i j}^{*}\right) \cdot s_{i j}^{*}=-1.2$ and $\left(1-F_{i j}^{*}\right) \cdot s_{i j}^{*}=-2.7$. Because $\min \left\{N\left(T_{i j}^{*}\right.\right.$. $\left.\left.s_{i j}^{*}\right), N\left(\left(1-I_{i j}^{*}\right) \cdot s_{i j}^{*}\right), N\left(\left(1-F_{i j}^{*}\right) \cdot s_{i j}^{*}\right)\right\}=\min (-0.3,-1.2,-2.7)=2.7 \in[-3,-2]$, then $a=-3$ and $\left(s_{i j}^{*},<T_{i j}^{*}, I_{i j}^{*}, F_{i j}^{*}>\right)=\left(s_{-3},<0.1,0.6,0.1>\right)$.

(3) Besides, there are two other situations: one is that the value of $N\left(T_{i j}^{*} \cdot s_{i j}^{*}\right), N\left(\left(1-I_{i j}^{*}\right) \cdot s_{i j}^{*}\right)$ and $N\left(\left(1-F_{i j}^{*}\right) \cdot s_{i j}^{*}\right)$ are a positive number and two negative numbers; the other one is that there are two negative numbers and a positive number among $N\left(T_{i j}^{*} \cdot s_{i j}^{*}\right), N\left(\left(1-I_{i j}^{*}\right) \cdot s_{i j}^{*}\right)$ and $N\left(\left(1-F_{i j}^{*}\right) \cdot s_{i j}^{*}\right)$. In these conditions, the final answers may not meet the requirements of $0 \leq T_{i j}^{*} \leq 1,0 \leq 1-I_{i j}^{*} \leq 1$ or $0 \leq 1-F_{i j}^{*} \leq 1$. In other words, the consistent matrix being obtained may not be a SNLPR. But it still does not affect us to measure the consistency degree of the SNLPR, for the reason that the values of $T_{i j}^{*} \cdot s_{i j^{\prime}}^{*}\left(1-I_{i j}^{*}\right) \cdot s_{i j}^{*}$ and $\left(1-F_{i j}^{*}\right) \cdot s_{i j}^{*}$ can be calculated. Thus, the consistency index of the SNLPR can be acquired (more details see Definition 12, Definition 17 and Definition 18).

Example 5. Suppose the same SNLPR in Example 2 is given. A consistent SNLPR $K_{1}^{*}=$ $\left[\begin{array}{ccc}\left(s_{0},<1,0,0>\right) & \left(s_{1},<0.133,0.433,0.167>\right) & \left(s_{1},<0.067,0.567,0.934>\right) \\ \left(s_{-1},<0.133,0.433,0.167>\right) & \left(s_{0},<1,0,0>\right) & \left(s_{-1},<0.067,0.867,0.433>\right) \\ \left(s_{-1},<0.067,0.567,0.934>\right) & \left(s_{1},<0.067,0.867,0.433>\right) & \left(s_{0},<1,0,0>\right)\end{array}\right]$ can be
obtained in the abovementioned way.

According to those distance measures of SNLNs in Section 3, several distance measures of SNLPRs are further defined.

Definition 17. There are two facultative SNLPRs $A=\left(a_{i j}\right)_{n \times n}$ and $B=\left(b_{i j}\right)_{n \times n^{\prime}}$ the linguistic term set is $S=\left\{s_{i} \mid i \in[-g, g]\right\}$. Then Hamming distance $D_{H}(A, B)$, Euclidean distance $D_{E}(A, B)$ and Hausdorff distance $D_{\mathrm{Ha}}(A, B)$ between $A$ and $B$ are defined as:

$$
\begin{aligned}
D_{H}(A, B) & =\frac{1}{n(n-1)} \sum_{i \neq j}^{n} d_{H}\left(a_{i j}, b_{i j}\right) \\
D_{E}(A, B) & =\frac{1}{n(n-1)} \sum_{i \neq j}^{n} d_{E}\left(a_{i j}, b_{i j}\right) \\
D_{H a}(A, B) & =\frac{1}{n(n-1)} \sum_{i \neq j}^{n} d_{H a}\left(a_{i j}, b_{i j}\right) .
\end{aligned}
$$

Example 6. Assume $g=4$, and two SNLPR $A=\left[\begin{array}{ccc}\left(s_{0},<1,0,0>\right) & \left(s_{1},<02,0.3,0.6>\right) & \left(s_{3},<0.5,0.4,0.2>\right) \\ \left(s_{-1},<0.2,0.3,0.6>\right) & \left(s_{0},<1,0,0>\right) & \left(s_{-2}<0.7,0.1,0.6>\right) \\ \left(s_{-3},<0.5,0.4,0.2>\right) & \left(s_{2},<0.7,0.1,0.6>\right) & \left(s_{0},<1,0,0>\right)\end{array}\right]$, $B=\left[\begin{array}{ccc}\left(s_{0},<1,0,0>\right) & \left(s_{2},<0.5,0.1,0.4>\right) & \left(s_{3},<0.3,0.6,0.1>\right) \\ \left(s_{-2}<0.5,0.1,0.4>\right) & \left(s_{0},<1,0,0>\right) & \left(s_{-1},<0.2,0.8,0.5>\right) \\ \left(s_{-3},<0.3,0.6,0.1>\right) & \left(s_{1},<0.2,0.8,0.5>\right) & \left(s_{0},<1,0,0>\right)\end{array}\right]$. Then Hamming distance $D_{H}(A, B) \approx 0.1014$, Euclidean distance $D_{E}(A, B) \approx 0.1083$ and Hausdorff distance $D_{H a}(A, B) \approx 0.1375$.

Theorem 3. Given two SNLPRs $A=\left(a_{i j}\right)_{n \times n}$ and $B=\left(b_{i j}\right)_{n \times n^{\prime}}$ if $D_{H}(A, B), D_{E}(A, B)$ and $D_{H a}(A, B)$ can satisfy the following properties:

(1) $0 \leq D_{H}(A, B) \leq 1,0 \leq D_{E}(A, B) \leq 1$, and $0 \leq D_{H a}(A, B) \leq 1$;

(2) $D_{H}(A, B)=D_{H}(B, A), D_{E}(A, B)=D_{E}(B, A)$, and $D_{H a}(A, B)=D_{H a}(B, A)$;

(3) If $A=B$, then $D_{H}(A, B)=0, D_{E}(A, B)=0$, and $D_{H a}(A, B)=0$; 
(4) Let $A=\left(a_{i j}\right)_{n \times n}=\left(s a_{i j},<T_{i j}^{a}, I_{i j}^{a}, F_{i j}^{a}>\right)_{n \times n^{\prime}} B=\left(b_{i j}\right)_{n \times n}=\left(s b_{i j},<T_{i j}^{b}, I_{i j}^{b}, F_{i j}^{b}>\right)_{n \times n}$ and $C=\left(c_{i j}\right)_{n \times n}=\left(s c_{i j},<T_{i j}^{c}, I_{i j}^{c}, F_{i j}^{c}>\right)_{n \times n}$ be three SNLPRs, if $s_{0} \leq s a_{i j} \leq s b_{i j} \leq s c_{i j}, T_{i j}^{a} \leq T_{i j}^{b} \leq T_{i j}^{c}$ $I_{i j}^{a} \geq I_{i j}^{b} \geq I_{i j}^{c}$ and $F_{i j}^{a} \geq F_{i j}^{b} \geq F_{i j}^{c}$ for all $i, j=1,2, \cdots n$, then $D_{H}(A, B) \leq D_{H}(A, C)$, $D_{H}(B, C) \leq D_{H}(A, C), D_{E}(A, B) \leq D_{E}(A, C), D_{E}(B, C) \leq D_{E}(A, C), D_{H a}(A, B) \leq D_{H a}(A, C)$ and $D_{\mathrm{Ha}}(B, C) \leq D_{\mathrm{Ha}}(A, C)$.

\section{Proof 4.}

(1) Since $0 \leq d_{H}\left(a_{i j}, b_{i j}\right) \leq 1 \Rightarrow 0 \leq \frac{1}{n(n-1)} \sum_{i \neq j}^{n} d_{H}\left(a_{i j}, b_{i j}\right) \leq 1$, then $0 \leq D_{H}(A, B) \leq 1$.

Likewise, $0 \leq D_{E}(A, B) \leq 1$ and $0 \leq D_{H a}(A, B) \leq 1$.

(2) As $d_{H}\left(a_{i j}, b_{i j}\right)=d_{H}\left(b_{i j}, a_{i j}\right) \Rightarrow \frac{1}{n(n-1)} \sum_{i \neq j}^{n} d_{H}\left(a_{i j}, b_{i j}\right)=\frac{1}{n(n-1)} \sum_{i \neq j}^{n} d_{H}\left(b_{i j}, a_{i j}\right)$, then $D_{H}(A, B)=$ $D_{H}(B, A)$. Similarly, $D_{E}(A, B)=D_{E}(B, A)$ and $D_{H a}(A, B)=D_{H a}(B, A)$.

(3) Because $A=B$, for all $i, j=1,2, \cdots n$, then $a_{i j}=b_{i j} \Rightarrow d_{H}\left(a_{i j}, b_{i j}\right)=0 \Rightarrow D_{H}(A, B)=$ $\frac{1}{n(n-1)} \sum_{i \neq j}^{n} d_{H}\left(a_{i j}, b_{i j}\right)=0$. In the same way, $D_{E}(A, B)=0$ and $D_{H a}(A, B)=0$.

(4) As $d_{H}\left(a_{i j}, b_{i j}\right) \leq d_{H}\left(a_{i j}, c_{i j}\right) \Rightarrow \frac{1}{n(n-1)} \sum_{i \neq j}^{n} d_{H}\left(a_{i j}, b_{i j}\right) \leq \frac{1}{n(n-1)} \sum_{i \neq j}^{n} d_{H}\left(a_{i j}, c_{i j}\right) \Rightarrow D_{H}\left(a_{i j}, b_{i j}\right) \leq$ $D_{H}\left(a_{i j}, c_{i j}\right)$. Similarly, $D_{H}\left(b_{i j}, c_{i j}\right) \leq D_{H}\left(a_{i j}, c_{i j}\right), D_{E}\left(a_{i j}, b_{i j}\right) \leq D_{E}\left(a_{i j}, c_{i j}\right), D_{E}\left(b_{i j}, c_{i j}\right) \leq$ $D_{E}\left(a_{i j}, c_{i j}\right), D_{H a}\left(a_{i j}, b_{i j}\right) \leq D_{H a}\left(a_{i j}, c_{i j}\right)$ and $D_{H a}\left(b_{i j}, c_{i j}\right) \leq D_{H a}\left(a_{i j}, c_{i j}\right)$.

This is the end of Proof 4.

Definition 18. Let $K=\left(k_{i j}\right)_{n \times n}$ be a SNLPR, and $K^{*}=\left(k_{i j}^{*}\right)_{n \times n}$ be the corresponding consistent SNLPR, the deviation between $K$ and $K^{*}$ can be expressed by a consistency index $C(K)$ as follows:

$$
C X(K)=1-D\left(K, K^{*}\right),
$$

where $D\left(K, K^{*}\right)$ can be replaced by $D_{H}\left(K, K^{*}\right), D_{E}\left(K, K^{*}\right)$, or $D_{H a}\left(K, K^{*}\right)$.

Example 7. Assume a SNLPR $K_{1}=\left[\begin{array}{ccc}\left(s_{0},<1,0,0>\right) & \left(s_{-1},<0.4,0.2,0.1>\right) & \left(s_{2},<0.3,0.1,0.2>\right) \\ \left(s_{1},<0.4,0.2,0.1>\right) & \left(s_{0},<1,0,0>\right) & \left(s_{-3},<0.2,0.5,0.3>\right) \\ \left(s_{-2},<0.3,0.1,0.2>\right) & \left(s_{3},<0.2,0.5,0.3>\right) & \left(s_{0},<1,0,0>\right)\end{array}\right]$ is the same with Example 2, and its consistent SNLPR is $K_{1}^{*}=$ $\left[\begin{array}{ccc}\left(s_{0},<1,0,0>\right) & \left(s_{1},<0.133,0.433,0.167>\right) & \left(s_{1},<0.067,0.567,0.934>\right) \\ \left(s_{-1},<0.133,0.433,0.167>\right) & \left(s_{0},<1,0,0>\right) & \left(s_{-1},<0.067,0.867,0.433>\right) \\ \left(s_{-1},<0.067,0.567,0.934>\right) & \left(s_{1},<0.067,0.867,0.433>\right) & \left(s_{0},<1,0,0>\right)\end{array}\right]$ from Example 5. If $D_{H}\left(K, K^{*}\right)$ is used, then $C X(K) \approx 0.8569$; if $D_{E}\left(K, K^{*}\right)$ is used, then $C X(K) \approx 0.9936$; if $D_{H a}\left(K, K^{*}\right)$ is used, then $C X(K) \approx 0.8083$.

Note that since $0 \leq D\left(K, K^{*}\right) \leq 1$, then $0 \leq C X(K) \leq 1$. Moreover, the greater the value of $C X(K)$, the more consistent $K$ will be according to Definition 18 .

\subsection{Improving the Consistency of SNLPRs}

Normally, it is difficult for DMs to provide a fully consistent SNLPR. There will be a lot of uncertainty in the decision-making process. For this reason, it is appropriate and necessary to allow the SNLPR presented by DMs satisfy the consistency in some extent. Then, the following is the concept of acceptable consistency.

Definition 19. Let $C X$ be a consistency threshold value. For an arbitrary SNLPR $K$, if the corresponding consistency index is $C X(K)$, and

$$
C X(K)>C X,
$$

then $\mathrm{K}$ is consistent in some extent. In other words, it has acceptable consistency. 
Zhu and $\mathrm{Xu}[74]$ indicated that the consistency index $C X(K)$ obeys a normal distribution, thus providing a method to determine the consistency threshold value $C X$. This method is used here. When the significance level $\alpha=0.1$ and the standard deviation $\sigma=0.2$, the consistency index threshold is shown in Table 1.

Table 1. The consistency threshold value $C X$.

\begin{tabular}{llllllll}
\hline $\boldsymbol{C X}$ & $\boldsymbol{n}=\mathbf{3}$ & $\boldsymbol{n}=\mathbf{4}$ & $\boldsymbol{n}=\mathbf{5}$ & $\boldsymbol{n}=\mathbf{6}$ & $\boldsymbol{n}=\mathbf{7}$ & $\boldsymbol{n}=\mathbf{8}$ & $\boldsymbol{n}=\mathbf{9}$ \\
\hline$g=2$ & 0.8235 & 0.7576 & 0.7210 & 0.6981 & 0.6824 & 0.6710 & 0.6624 \\
$g=3$ & 0.8739 & 0.8269 & 0.8007 & 0.7844 & 0.7731 & 0.7650 & 0.7589 \\
$g=4$ & 0.9020 & 0.8653 & 0.8450 & 0.8323 & 0.8235 & 0.8172 & 0.8124 \\
\hline
\end{tabular}

Of course, the numbers in Table 1 can be used for reference. DMs can determine the value of thresholds based on their previous experience, preferences, or actual situations as well.

Example 8. Suppose a SNLPR $K_{1}$ is the same as Example 2, and the consistency index $C X\left(K_{1}\right) \approx 0.8569$ from Example 7. If $D_{H}\left(K_{1}, K_{1}^{*}\right)$ is used, for $g=4$ and $n=3$, the consistency threshold value can be assigned with $C X=0.9020$ based on Table $1 . C X\left(K_{1}\right)<C X$, and it demonstrates that $K_{1}$ does not have acceptable consistency.

When the initial SNLPR presented by DMs is not acceptably consistent, a way to improve this SNLPR should be provided. Then, an iterative algorithm (Algorithm 1) is given to achieve acceptable consistency as follows:

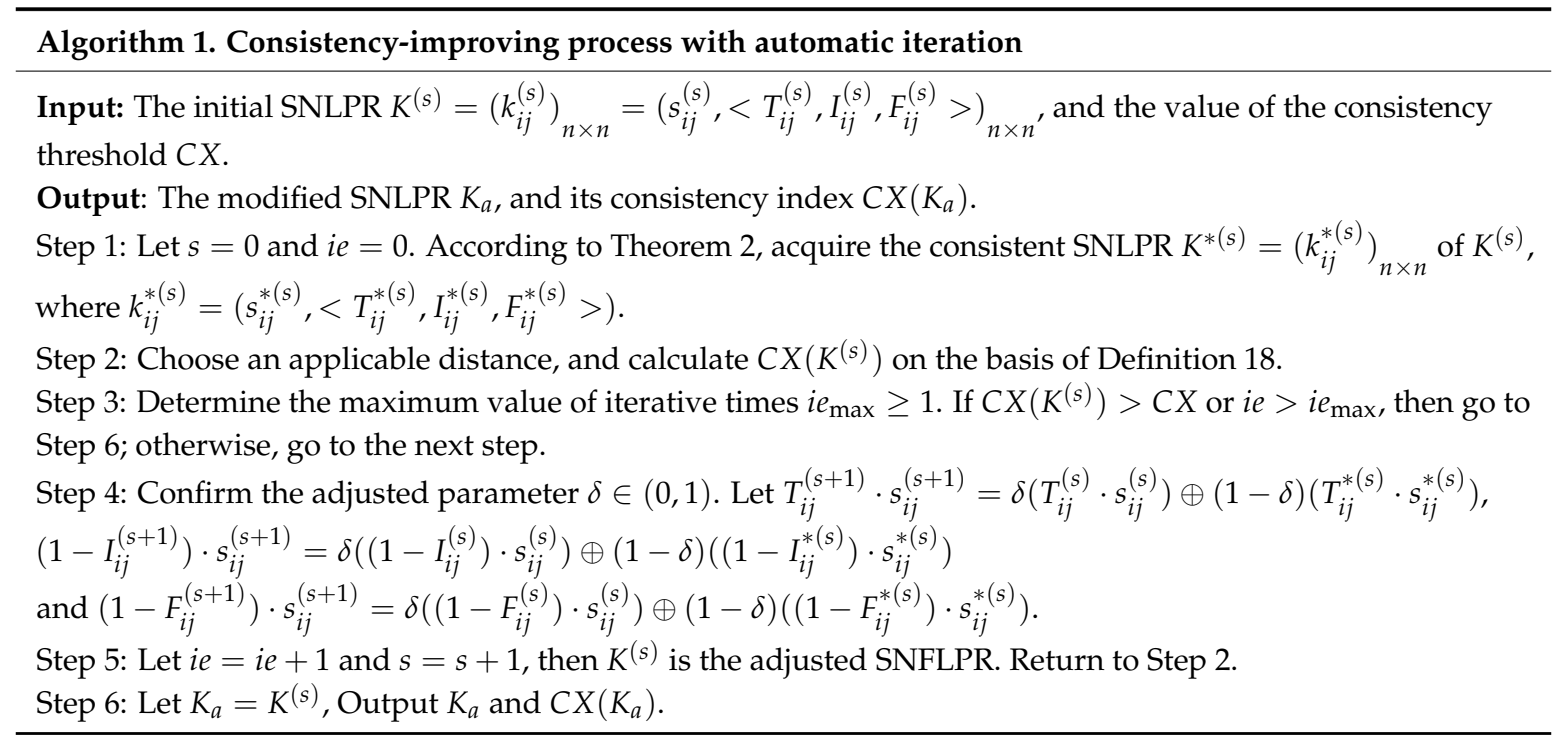

This algorithm above improves the consistency through the iterative process automatically, which is convenient and efficient.

Theorem 4. Given a SNLPR $K$, if $K$ does not have acceptable consistency, it will be more consistent using Algorithm 1. That is to say, $C\left(K^{(s+1)}\right)<C\left(K^{(s)}\right)$ is true. Moreover, $\lim _{s \rightarrow \infty} C\left(K^{(s)}\right)=0$.

\section{Proof 5.}

(1) From Equation (18), $T_{i j}^{*(s)} \cdot s_{i j}^{*(s)}=\frac{1}{n}\left\{\oplus_{k=1}^{n}\left[\left(T_{i k}^{(s)} \cdot s_{i k}^{(s)}\right) \oplus\left(T_{k j}^{(s)} \cdot s_{k j}^{(s)}\right)\right]\right\}$, and then $\left|\mathrm{T}_{\mathrm{ij}}^{(s+1)} \mathrm{s}_{i j}^{(s+1)}-\mathrm{T}_{\mathrm{ij}}^{*(s+1)} \mathrm{s}_{i j}^{*(s+1)}\right|=\left|T_{i j}^{(s+1)} s_{i j}^{(s+1)}-\frac{1}{n}\left\{\oplus_{k=1}^{n}\left[\left(T_{i k}^{(s+1)} \cdot s_{i k}^{(s+1)}\right) \oplus\left(T_{k j}^{(s+1)} \cdot s_{k j}^{(s+1)}\right)\right]\right\}\right|=$ $\mid \delta\left(T_{i j}^{(s)} \cdot s_{i j}^{(s)}\right) \oplus(1-\delta)\left(T_{i j}^{*(s)} \cdot s_{i j}^{*(s)}\right)-\frac{1}{n}\left\{\oplus_{k=1}^{n}\left[\left(\delta\left(T_{i k}^{(s)} \cdot s_{i k}^{(s)}\right) \oplus(1-\delta)\left(T_{i k}^{*(s)} \cdot s_{i k}^{*(s)}\right)\right) \oplus\left(\delta\left(T_{k j}^{(s)}\right.\right.\right.\right.$. 
$\left.\left.\left.\left.s_{k j}^{(s)}\right) \oplus(1-\delta)\left(T_{k j}^{*(s)} \cdot s_{k j}^{*(s)}\right)\right)\right]\right\}|\leq| \delta\left(T_{i j}^{(s)} \cdot s_{i j}^{(s)}\right) \oplus-\frac{1}{n}\left\{\oplus_{k=1}^{n}\left[\left(\delta\left(T_{i k}^{(s)} \cdot s_{i k}^{(s)}\right) \oplus\left(\delta\left(T_{k j}^{(s)} \cdot s_{k j}^{(s)}\right)\right)\right]\right\}|+|(1-\right.$ $\left.\left.\delta)\left(T_{i j}^{*(s)} \cdot s_{i j}^{*(s)}\right)-\frac{1}{n}\left\{\oplus_{k=1}^{n}\left[(1-\delta)\left(T_{i k}^{*(s)} \cdot s_{i k}^{*(s)}\right)\right) \oplus(1-\delta)\left(T_{k j}^{*(s)} \cdot s_{k j}^{*(s)}\right)\right)\right]\right\} \mid$

$=\delta\left|\left(T_{i j}^{(s)} \cdot s_{i j}^{(s)}\right) \oplus-\frac{1}{n}\left\{\oplus_{k=1}^{n}\left[\left(T_{i k}^{(s)} \cdot s_{i k}^{(s)}\right) \oplus\left(T_{k j}^{(s)} \cdot s_{k j}^{(s)}\right)\right]\right\}\right|+(1-\delta) \mid\left(T_{i j}^{*(s)} \cdot s_{i j}^{*(s)}\right)-$ $\frac{1}{n}\left\{\oplus_{k=1}^{n}\left[\left(T_{i k}^{*(s)} \cdot s_{i k}^{*(s)}\right) \oplus\left(T_{k j}^{*(s)} \cdot s_{k j}^{*(s)}\right)\right]\right\}|=\delta|\left(T_{i j}^{(s)} \cdot s_{i j}^{(s)}\right) \oplus-T_{i j}^{*(s)} \cdot s_{i j}^{*(s)}|+|(1-\delta) \mid\left(T_{i j}^{*(s)}\right.$. $\left.s_{i j}^{*(s)}\right)-\frac{1}{n}\left\{\oplus_{k=1}^{n}\left[\left(\frac{1}{n}\left\{\oplus_{p=1}^{n}\left[\left(T_{i p}^{(s)} \cdot s_{i p}^{(s)}\right) \oplus\left(T_{p k}^{(s)} \cdot s_{p k}^{(s)}\right)\right]\right\}\right) \oplus\left(\frac{1}{n}\left\{\oplus_{p=1}^{n}\left[\left(T_{k p}^{(s)} \cdot s_{k p}^{(s)}\right) \oplus\left(T_{p j}^{(s)} \cdot s_{p j}^{(s)}\right)\right]\right\}\right)\right]\right\} \mid$ $=\delta\left|\left(T_{i j}^{(s)} \cdot s_{i j}^{(s)}\right) \oplus-T_{i j}^{*(s)} \cdot s_{i j}^{*(s)}\right|+(1-\delta)\left|\left(T_{i j}^{*(s)} \cdot s_{i j}^{*(s)}\right)-\left(\frac{1}{n}\left\{\oplus_{p=1}^{n}\left[\left(T_{i p}^{(s)} \cdot s_{i p}^{(s)}\right) \oplus\left(T_{p j}^{(s)} \cdot s_{p j}^{(s)}\right)\right]\right\}\right)\right|$ $=\delta\left|\left(T_{i j}^{(s)} \cdot s_{i j}^{(s)}\right) \oplus-T_{i j}^{*(s)} \cdot s_{i j}^{*(s)}\right|+(1-\delta)\left|\left(T_{i j}^{*(s)} \cdot s_{i j}^{*(s)}\right)-\left(T_{i j}^{*(s)} \cdot s_{i j}^{*(s)}\right)\right|=\delta \mid\left(T_{i j}^{(s)} \cdot s_{i j}^{(s)}\right) \oplus-T_{i j}^{*(s)}$. $s_{i j}^{*(s)} \mid$, so $N\left(\left|T_{i j}^{(s+1)} s_{i j}^{(s+1)}-T_{i j}^{*(s+1)} s_{i j}^{*(s+1)}\right|\right) \leq \delta \cdot N\left(\left|\left(T_{i j}^{(s)} \cdot s_{i j}^{(s)}\right) \oplus-T_{i j}^{*(s)} \cdot s_{i j}^{*(s)}\right|\right)$;

(2) From Equation (19), $\left(1-I_{i j}^{*}\right) \cdot s_{i j}^{*}=\frac{1}{n}\left\{\oplus_{k=1}^{n}\left[\left(\left(1-I_{i k}\right) \cdot s_{i k}\right) \oplus\left(\left(1-I_{k j}\right) \cdot s_{k j}\right)\right]\right\}$, and then $\mid(1-$ $\left.I_{i j}^{(s+1)}\right) \cdot s_{i j}^{(s+1)}-\left(1-I_{i j}^{*(s+1)}\right) \cdot s_{i j}^{*(s+1)}|=|\left(1-I_{i j}^{(s+1)}\right) \cdot s_{i j}^{(s+1)}-\frac{1}{n}\left\{\oplus_{k=1}^{n}\left[\left(\left(1-I_{i k}^{(s+1)}\right) \cdot s_{i k}^{(s+1)}\right) \oplus\right.\right.$ $\left.\left.\left(\left(1-I_{k j}^{(s+1)}\right) \cdot s_{k j}^{(s+1)}\right)\right]\right\}|=| \delta\left(\left(1-I_{i j}^{(s)}\right) \cdot s_{i j}^{(s)}\right) \oplus(1-\delta)\left(\left(1-I_{i j}^{*(s)}\right) \cdot s_{i j}^{*(s)}\right)-\frac{1}{n}\left\{\oplus_{k=1}^{n}\left[\left(\delta\left(\left(1-I_{i k}^{(s)}\right) \cdot\right.\right.\right.\right.$ $\left.\left.\left.\left.s_{i k}^{(s)}\right) \oplus(1-\delta)\left(\left(1-I_{i k}^{*(s)}\right) \cdot s_{i k}^{*(s)}\right)\right) \oplus\left(\delta\left(\left(1-I_{i j}^{(s)}\right) \cdot s_{k j}^{(s)}\right) \oplus(1-\delta)\left(\left(1-I_{k j}^{*(s)}\right) \cdot s_{k j}^{*(s)}\right)\right)\right]\right\} \mid$ $\leq \mid \delta\left(\left(1-I_{i j}^{(s)}\right) \cdot s_{i j}^{(s)}\right)-\frac{1}{n}\left\{\oplus_{k=1}^{n}\left[\left(\delta\left(\left(1-I_{i k}^{(s)}\right) \cdot s_{i k}^{(s)}\right) \oplus\left(\delta\left(\left(1-I_{k j}^{(s)}\right) \cdot s_{k j}^{(s)}\right)\right)\right]\right\} \mid+\right.$ $\mid(1-\delta)\left(\left(1-I_{i j}^{*(s)}\right) \cdot s_{i j}^{*(s)}\right)-\frac{1}{n}\left\{\oplus_{k=1}^{n}\left[(1-\delta)\left(\left(1-I_{i k}^{*(s)}\right) \cdot s_{i k}^{*(s)}\right)\right) \oplus_{k=1}^{n}(1-\delta)\left(\left(1-I_{k j}^{*(s)}\right) \cdot\right.\right.$ $\left.\left.\left.\left.\left.s_{k j}^{*(s)}\right)\right)\right]\right\}|=\delta|\left(\left(1-I_{i j}^{(s)}\right) \cdot s_{i j}^{(s)}\right)-\frac{1}{n}\left\{\oplus_{k=1}^{n}\left[\left(\left(1-I_{i k}^{(s)}\right) \cdot s_{i k}^{(s)}\right) \oplus\left(\left(1-I_{k j}^{(s)}\right) \cdot s_{k j}^{(s)}\right)\right)\right]\right\}|+(1-\delta)|((1-$ $\left.\left.\left.\left.I_{i j}^{*(s)}\right) \cdot s_{i j}^{*(s)}\right)-\frac{1}{n}\left\{\oplus_{k=1}^{n}\left[\left(\left(1-I_{i k}^{*(s)}\right) \cdot s_{i k}^{*(s)}\right)\right) \oplus_{k=1}^{n}\left(\left(1-I_{k j}^{*(s)}\right) \cdot s_{k j}^{*(s)}\right)\right)\right]\right\} \mid$

$=\delta\left|\left(\left(1-I_{i j}^{(s)}\right) \cdot s_{i j}^{(s)}\right)-\left(1-I_{i j}^{*(s)}\right) \cdot s_{i j}^{*(s)}\right|+(1-\delta) \mid\left(\left(1-I_{i j}^{*(s)}\right) \cdot s_{i j}^{*(s)}\right)-\frac{1}{n}\left\{\oplus_{k=1}^{n}\left[\left(\frac{1}{n}\left\{\oplus_{p=1}^{n}[((1-\right.\right.\right.\right.$ $\left.\left.I_{i p}^{(s)}\right) \cdot s_{i p}^{(s)}\right) \oplus$

$\left.\left.\left.\left.\left.\left(\left(1-I_{p k}^{(s)}\right) \cdot s_{p k}^{(s)}\right)\right]\right\}\right) \oplus_{k=1}^{n}\left(\frac{1}{n}\left\{\oplus_{p=1}^{n}\left[\left(\left(1-I_{k p}^{(s)}\right) \cdot s_{k p}^{(s)}\right) \oplus\left(\left(1-I_{p j}^{(s)}\right) \cdot s_{p j}^{(s)}\right)\right]\right\}\right)\right]\right\} \mid$

$=\delta\left|\left(\left(1-I_{i j}^{(s)}\right) \cdot s_{i j}^{(s)}\right)-\left(1-I_{i j}^{*(s)}\right) \cdot s_{i j}^{*(s)}\right|+(1-\delta) \mid\left(\left(1-I_{i j}^{*(s)}\right) \cdot s_{i j}^{*(s)}\right)-\frac{1}{n}\left\{\oplus_{p=1}^{n}\left[\left(\left(1-I_{i p}^{(s)}\right) \cdot s_{i p}^{(s)}\right) \oplus\right.\right.$ $\left.\left.\left.\left(\left(1-I_{p j}^{(s)}\right) \cdot s_{p j}^{(s)}\right)\right]\right\}|=\delta|\left(1-I_{i j}^{(s)}\right) \cdot s_{i j}^{(s)}\right)-\left(1-I_{i j}^{*(s)}\right) \cdot s_{i j}^{*(s)}|+(1-\delta)|\left(1-I_{i j}^{*(s)}\right) \cdot s_{i j}^{*(s)}-\left(1-I_{i j}^{*(s)}\right) \cdot$ $s_{i j}^{*(s)}|=\delta|\left(1-I_{i j}^{(s)}\right) \cdot s_{i j}^{(s)}-\left(1-I_{i j}^{*(s)}\right) \cdot s_{i j}^{*(s)} \mid$, so $N\left(\left(1-I_{i j}^{(s+1)}\right) \cdot s_{i j}^{(s+1)}-\left(1-I_{i j}^{*(s+1)}\right) \cdot s_{i j}^{*(s+1)} \mid\right) \leq$ $\delta \cdot N\left(\left|\left(1-I_{i j}^{(s)}\right) \cdot s_{i j}^{(s)}-\left(1-I_{i j}^{*(s)}\right) \cdot s_{i j}^{*(s)}\right|\right)$;

(3) From Equation (20), $\left(1-F_{i j}^{*}\right) \cdot s_{i j}^{*}=\frac{1}{n}\left\{\oplus_{k=1}^{n}\left[\left(\left(1-F_{i k}\right) \cdot s_{i k}\right) \oplus\left(\left(1-F_{k j}\right) \cdot s_{k j}\right)\right]\right\}$, and then $\mid(1-$ $\left.F_{i j}^{(s+1)}\right) \cdot s_{i j}^{(s+1)}-\left(1-F_{i j}^{*(s+1)}\right) \cdot s_{i j}^{*(s+1)}|=|\left(1-F_{i j}^{(s+1)}\right) \cdot s_{i j}^{(s+1)}-\frac{1}{n}\left\{\oplus_{k=1}^{n}\left[\left(\left(1-F_{i k}^{(s+1)}\right) \cdot s_{i k}^{(s+1)}\right) \oplus\right.\right.$ $\left.\left.\left(\left(1-F_{k j}^{(s+1)}\right) \cdot s_{k j}^{(s+1)}\right)\right]\right\}|=| \delta\left(\left(1-F_{i j}^{(s)}\right) \cdot s_{i j}^{(s)}\right) \oplus(1-\delta)\left(\left(1-F_{i j}^{*(s)}\right) \cdot s_{i j}^{*(s)}\right)-\frac{1}{n}\left\{\oplus_{k=1}^{n}\left[\left(\delta\left(\left(1-F_{i k}^{(s)}\right)\right.\right.\right.\right.$. $\left.\left.\left.\left.s_{i k}^{(s)}\right) \oplus(1-\delta)\left(\left(1-F_{i k}^{*(s)}\right) \cdot s_{i k}^{*(s)}\right)\right) \oplus\left(\delta\left(\left(1-F_{i j}^{(s)}\right) \cdot s_{k j}^{(s)}\right) \oplus(1-\delta)\left(\left(1-F_{k j}^{*(s)}\right) \cdot s_{k j}^{*(s)}\right)\right)\right]\right\} \mid$ $\leq \mid \delta\left(\left(1-F_{i j}^{(s)}\right) \cdot s_{i j}^{(s)}\right)-\frac{1}{n}\left\{\oplus_{k=1}^{n}\left[\left(\delta\left(\left(1-F_{i k}^{(s)}\right) \cdot s_{i k}^{(s)}\right) \oplus\left(\delta\left(\left(1-F_{k j}^{(s)}\right) \cdot s_{k j}^{(s)}\right)\right)\right]\right\} \mid+\right.$ $\left.\left.\mid(1-\delta)\left(\left(1-F_{i j}^{*(s)}\right) \cdot s_{i j}^{*(s)}\right)-\frac{1}{n}\left\{\oplus_{k=1}^{n}\left[(1-\delta)\left(\left(1-F_{i k}^{*(s)}\right) \cdot s_{i k}^{*(s)}\right)\right) \oplus_{k=1}^{n}(1-\delta)\left(\left(1-F_{k j}^{*(s)}\right) \cdot s_{k j}^{*(s)}\right)\right)\right]\right\} \mid$ $\left.=\delta \mid\left(\left(1-F_{i j}^{(s)}\right) \cdot s_{i j}^{(s)}\right)-\frac{1}{n}\left\{\oplus_{k=1}^{n}\left[\left(\left(1-F_{i k}^{(s)}\right) \cdot s_{i k}^{(s)}\right) \oplus\left(\left(1-F_{k j}^{(s)}\right) \cdot s_{k j}^{(s)}\right)\right)\right]\right\}|+(1-\delta)|\left(\left(1-F_{i j}^{*(s)}\right) \cdot\right.$ $\left.\left.\left.s_{i j}^{*(s)}\right)-\frac{1}{n}\left\{\oplus_{k=1}^{n}\left[\left(\left(1-F_{i k}^{*(s)}\right) \cdot s_{i k}^{*(s)}\right)\right) \oplus_{k=1}^{n}\left(\left(1-F_{k j}^{*(s)}\right) \cdot s_{k j}^{*(s)}\right)\right)\right]\right\} \mid$ $=\delta\left|\left(\left(1-F_{i j}^{(s)}\right) \cdot s_{i j}^{(s)}\right)-\left(1-F_{i j}^{*(s)}\right) \cdot s_{i j}^{*(s)}\right|+(1-\delta) \mid\left(\left(1-F_{i j}^{*(s)}\right) \cdot s_{i j}^{*(s)}\right)-\frac{1}{n}\left\{\oplus_{k=1}^{n}\left[\left(\frac{1}{n}\left\{\oplus_{p=1}^{n}[((1-\right.\right.\right.\right.$ $\left.\left.F_{i p}^{(s)}\right) \cdot s_{i p}^{(s)}\right) \oplus$

$\left.\left.\left.\left.\left.\left(\left(1-F_{p k}^{(s)}\right) \cdot s_{p k}^{(s)}\right)\right]\right\}\right) \oplus_{k=1}^{n}\left(\frac{1}{n}\left\{\oplus_{p=1}^{n}\left[\left(\left(1-F_{k p}^{(s)}\right) \cdot s_{k p}^{(s)}\right) \oplus\left(\left(1-F_{p j}^{(s)}\right) \cdot s_{p j}^{(s)}\right)\right]\right\}\right)\right]\right\} \mid$ 
$=\delta\left|\left(\left(1-F_{i j}^{(s)}\right) \cdot s_{i j}^{(s)}\right)-\left(1-F_{i j}^{*(s)}\right) \cdot s_{i j}^{*(s)}\right|+(1-\delta) \mid\left(\left(1-F_{i j}^{*(s)}\right) \cdot s_{i j}^{*(s)}\right)-\frac{1}{n}\left\{\oplus_{p=1}^{n}[((1-\right.$ $\left.\left.\left.\left.\left.F_{i p}^{(s)}\right) \cdot s_{i p}^{(s)}\right) \oplus\left(\left(1-F_{p j}^{(s)}\right) \cdot s_{p j}^{(s)}\right)\right]\right\}|=\delta|\left(1-F_{i j}^{(s)}\right) \cdot s_{i j}^{(s)}\right)-\left(1-F_{i j}^{*(s)}\right) \cdot s_{i j}^{*(s)}|+(1-\delta)|(1-$ $\left.F_{i j}^{*(s)}\right) \cdot s_{i j}^{*(s)}-\left(1-F_{i j}^{*(s)}\right) \cdot s_{i j}^{*(s)}|=\delta|\left(1-F_{i j}^{(s)}\right) \cdot s_{i j}^{(s)}-\left(1-F_{i j}^{*(s)}\right) \cdot s_{i j}^{*(s)} \mid$, so $N\left(\left(1-F_{i j}^{(s+1)}\right)\right.$. $\left.s_{i j}^{(s+1)}-\left(1-F_{i j}^{*(s+1)}\right) \cdot s_{i j}^{*(s+1)} \mid\right) \leq \delta \cdot N\left(\left|\left(1-F_{i j}^{(s)}\right) \cdot s_{i j}^{(s)}-\left(1-F_{i j}^{*(s)}\right) \cdot s_{i j}^{*(s)}\right|\right)$; According to (1)-(3), there is $N\left(\left|T_{i j}^{(s+1)} s_{i j}^{(s+1)}-T_{i j}^{*(s+1)} s_{i j}^{*(s+1)}\right|\right)+N\left(\left(1-I_{i j}^{(s+1)}\right) \cdot s_{i j}^{(s+1)}-\left(1-I_{i j}^{*(s+1)}\right)\right.$. $\left.s_{i j}^{*(s+1)} \mid\right)+N\left(\left(1-F_{i j}^{(s+1)}\right) \cdot s_{i j}^{(s+1)}-\left(1-F_{i j}^{*(s+1)}\right) \cdot s_{i j}^{*(s+1)} \mid\right) \leq \delta \cdot\left[N\left(\mid\left(T_{i j}^{(s)} \cdot s_{i j}^{(s)}\right) \oplus-T_{i j}^{*(s)}\right.\right.$. $\left.\left.s_{i j}^{*(s)} \mid\right)+N\left(\left|\left(1-I_{i j}^{(s)}\right) \cdot s_{i j}^{(s)}-\left(1-I_{i j}^{*(s)}\right) \cdot s_{i j}^{*(s)}\right|\right)+N\left(\left|\left(1-F_{i j}^{(s)}\right) \cdot s_{i j}^{(s)}-\left(1-F_{i j}^{*(s)}\right) \cdot s_{i j}^{*(s)}\right|\right)\right]$, then $d_{H}\left(k_{i j}^{(s+1)}, k_{i j}^{*(s+1)}\right) \leq \delta d_{H}\left(k_{i j}^{(s)}, k_{i j}^{*(s)}\right), d_{E}\left(k_{i j}^{(s+1)}, k_{i j}^{*(s+1)}\right) \leq \delta d_{E}\left(k_{i j}^{(s)}, k_{i j}^{*(s)}\right), d_{H a}\left(k_{i j}^{(s+1)}, k_{i j}^{*(s+1)}\right) \leq$ $\delta d_{H a}\left(k_{i j}^{(s)}, k_{i j}^{*(s)}\right)$, so $C I\left(K^{(s+1)}\right)=1-D\left(K^{(s+1)}, K^{*(s+1)}\right)=\frac{1}{n(n-1)} \sum_{i \neq j}^{n} d\left(k_{i j}^{(s+1)}, k_{i j}^{*(s+1)}\right) \geq$ $\frac{1}{n(n-1)} \sum_{i \neq j}^{n} \delta \cdot d\left(k_{i j}^{(s)}, k_{i j}^{*(s)}\right) \geq C I\left(K^{(s)}\right)$. In addition, $\lim _{s \rightarrow \infty} C I\left(K^{(s)}\right)=1$.

This is the end of Proof 5.

It can be seen from Theorem 4 that an arbitrary SNLPR that does not have a satisfactory consistency can be adjusted by the above algorithm to an acceptable matrix. The value of the adjustment parameter will have an effect on the process speed and times. DMs or other experts can determine the value of $\delta$ based on the actual situation. In general, $\delta=0.5$ is advised. If the predetermined threshold is not satisfied, the algorithm will be repeated until the maximum number of iterations is reached.

Example 9. Let a SNLPR $K_{1}$ be the same as Example 2. It can be seen that $C X\left(K_{1}\right) \approx 0.8569$, and $K_{1}$ does not have acceptable consistency from Example 8. Then Algorithm 1 can be used to improve it.

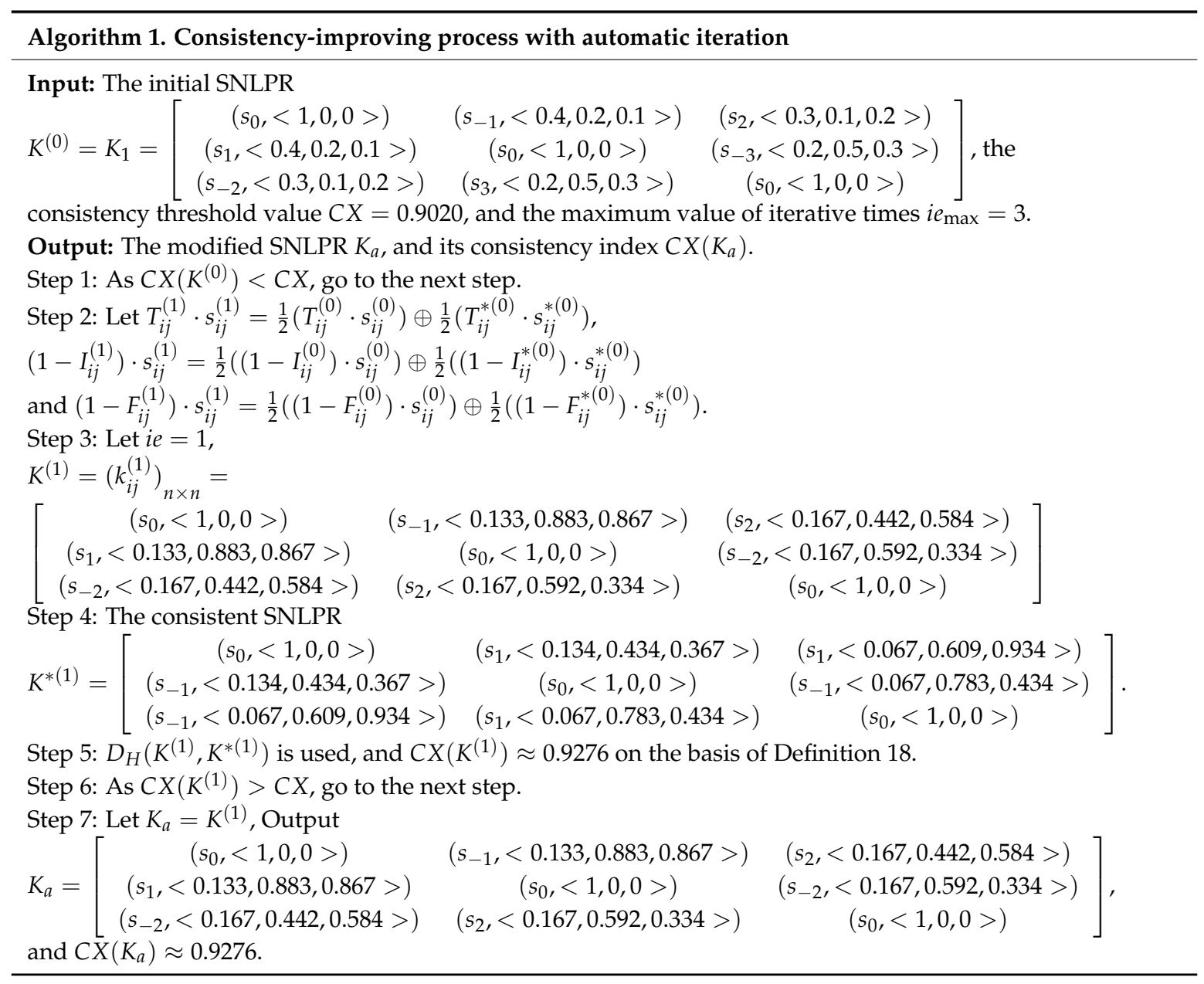




\subsection{A Decision-Making Approach with SNLPRs}

In this section, a decision-making method based on SNLPRs is presented.

Take a decision-making problem under simplified neutrosophic linguistic environment into consideration. Suppose there are a group of alternatives $X=\left\{x_{1}, x_{2}, \ldots, x_{n}\right\}$. The DMs want to get the ranking or select the eligible alternative from them. Then a preference matrix is formed after the linguistic term set $S=\left\{s_{i} \mid i \in[-g, g]\right\}$ is given. The basic elements in this matrix are SNLNs. Then the method based on SNLPRs is provided as Algorithm 2:

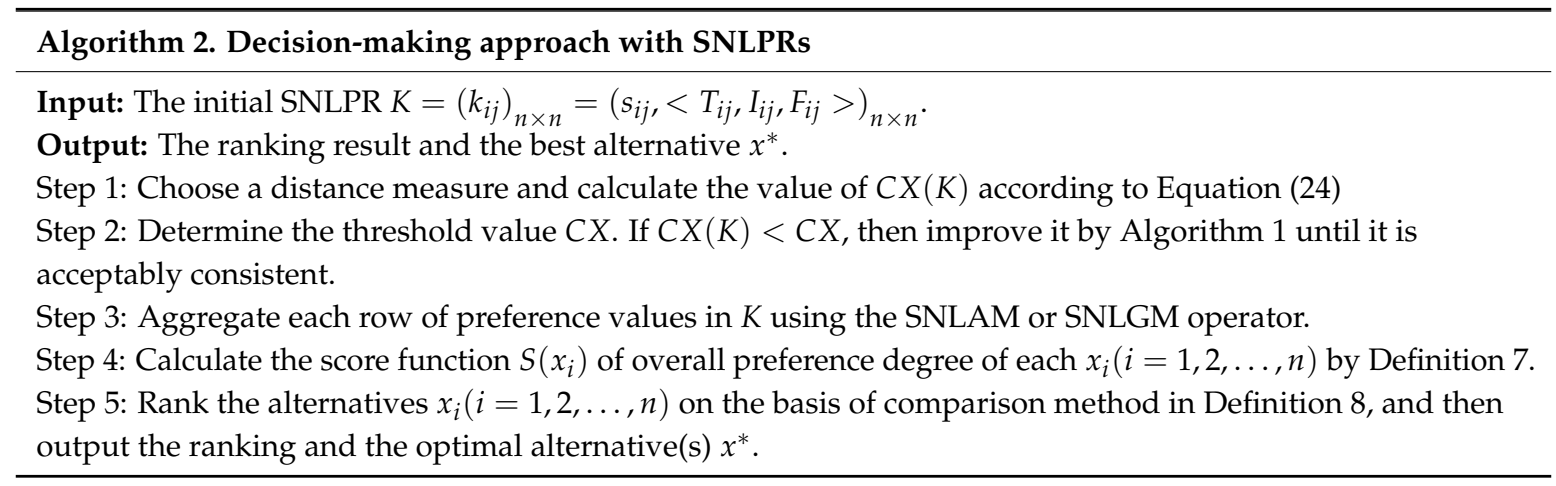

Note that it is a common and useful way to use aggregation operators to aggregate preference information, and then get the ranking result according to some comparison rules. However, Hou [75] pointed out that using arithmetic mean aggregation may get a reverse ranking. Therefore, the SNLGM operator to aggregate preference values is better.

\section{Application and Comparison}

The proposed decision-making method is applied to selecting project delivery models in this section. Some related comparison analyses are presented in the end.

YG Construction Co., Ltd. planned to select two suitable delivery models for a road construction project. The first one is chosen at once, and second one is reserved and accepted if necessary in the future. After a preliminary selection, four satisfactory options, DB, DBB, DBO, and CM, denoted by $\left\{x_{1}, x_{2}, x_{3}, x_{4}\right\}$, respectively, are considered. In order to pick out the right model, the project manager invites two experts to make evaluations together.

According to the properties of this project, the actual environment and the capability of owners, the project manager evaluates four alternatives. He presents his preference information with linguistic values. The linguistic term set used is $S=\left\{s_{i} \mid i \in[-4,4]\right\}$, where $s=\left\{s_{-4}=\right.$ much poorer, $s_{-3}=$ a lot poorer, $s_{-2}=$ poorer, $s_{-1}=$ slightly poorer, $s_{0}=$ fair, $s_{1}=$ slightly better, $s_{2}=$ better, $s_{3}=$ a lot better, $s_{4}=$ much better $\}$. Simultaneously, he gives the corresponding hesitant degrees of each preference value. Then two experts are asked to judge the possibility that the evaluation is inaccurate. In this way, SNLNs may be a good indication of their preference. As an example, the manager holds the view that $x_{1}$ is $s_{-1}$ to $x_{2}$, but he is not sure of his assessment. He thinks the degree of hesitation is 0.3. Afterwards, there is a probability of 0.9 that $s_{-1}$ is right, and a 0.2 probability of error given by two specialists. Therefore, they can be expressed by a SNLN $k_{12}=\left(s_{-1},<0.9,0.3,0.2>\right)$.

In the end, all the preference information yields an SNLPR as follows:

$$
K=\left[\begin{array}{cccc}
\left(s_{0},<1,0,0>\right) & \left(s_{-1},<0.9,0.3,0.2>\right) & \left(s_{1},<0.7,0.5,0.4>\right) & \left(s_{3},<0.8,0.3,0.1>\right) \\
\left(s_{1},<0.9,0.3,0.2>\right) & \left(s_{0},<1,0,0>\right) & \left(s_{1},<0.5,0.2,0.5>\right) & \left(s_{-2},<0.9,0.2,0.3>\right) \\
\left(s_{-1},<0.7,0.5,0.4>\right) & \left(s_{-1},<0.5,0.2,0.5>\right) & \left(s_{0},<1,0,0>\right) & \left(s_{-3},<0.6,0.7,0.1>\right) \\
\left(s_{-3},<0.8,0.3,0.1>\right) & \left(s_{2},<0.9,0.2,0.3>\right) & \left(s_{3},<0.6,0.7,0.1>\right) & \left(s_{0},<1,0,0>\right)
\end{array}\right] .
$$




\subsection{Illustration}

The decision-making method proposed in Section 4.4 is used to rank four options and select two models among them. The following are the specific steps:

Step 1: After discussion, DMs choose $D_{H}\left(K, K^{*}\right)$, and then calculate $C X(K) \approx 0.8628$ according to Equation (24).

Step 2: Because $g=4$ and $n=4$, DMs suggest the threshold value $C X=0.8653$ from Table 1 . And they find $C X(K)<C X$, then use Algorithm 1 to improve it as follows:

Let $K^{(0)}=K=\left(k_{i j}^{(0)}\right)_{n \times n}=\left(s_{i j}^{(0)},<T_{i j}^{(0)}, I_{i j}^{(0)}, F_{i j}^{(0)}>\right)_{n \times n}$ and $i e=0 . \quad$ According to Theorem 2, the consistent SNLPR $K^{*(0)}=\left(k_{i j}^{*(0)}\right)_{n \times n}=\left(s_{i j}^{*(0)},<T_{i j}^{*(0)}, I_{i j}^{*(0)}, F_{i j}^{*(0)}>\right)_{n \times n}=$ $\left[\begin{array}{cccc}\left(s_{0},<1,0,0>\right) & \left(s_{1},<0.65,0.5,0.35>\right) & \left(s_{2},<0.65,0.4875,0.2125>\right) & \left(s_{1},<0.25,0.625,0.725>\right) \\ \left(s_{-1},<0.65,0.5,0.35>\right) & \left(s_{0},<1,0,0>\right) & \left(s_{1},<0.65,0.475,0.075>\right) & \left(s_{-1},<0.4,0.875,0.625>\right) \\ \left(s_{-2},<0.65,0.4875,0.2125>\right) & \left(s_{-1},<0.65,0.475,0.075>\right) & \left(s_{0},<1,0,0>\right) & \left(s_{-2},<0.525,0.675,0.35>\right) \\ \left(s_{-1},<0.25,0.625,0.725>\right) & \left(s_{1},<0.4,0.875,0.625>\right) & \left(s_{2},<0.525,0.675,0.35>\right) & \left(s_{0},<1,0,0>\right)\end{array}\right]$. Since $C X\left(K^{(0)}\right)=C X(K) \approx 0.8649<C X$, determine $\delta=\frac{1}{2}$ and $i e_{\max }=3$. Let $K^{(1)}=K=\left(k_{i j}^{(1)}\right)_{n \times n}=\left(s_{i j}^{(1)},<T_{i j}^{(1)}, I_{i j}^{(1)}, F_{i j}^{(1)}>\right)_{n \times n}=$ $\left[\begin{array}{cccc}\left(s_{0},<1,0,0>\right) & \left(s_{-1},<0.125,0.9,0.925>\right) & \left(s_{2},<0.5,0.6188,0.4563>\right) & \left(s_{2},<0.66625,0.3813,0.2563>\right) \\ \left(s_{1},<0.125,0.9,0.925>\right) & \left(s_{0},<1,0,0>\right) & \left(s_{1},<0.575,0.3375,0.2875>\right) & \left(s_{-2}, 00.55,0.5688,0.5563>\right) \\ \left(s_{-2},<0.5,0.6188,0.4563>\right) & \left(s_{-1},<0.575,0.3375,0.2875>\right) & \left(s_{0},<1,0,0>\right) & \left(s_{-2}<0.7125,0.6125,0>\right) \\ \left(s_{-2},<0.6625,0.3813,0.2563>\right) & \left(s_{2},<0.55,0.5688,0.5563>\right) & \left(s_{2},<0.7125,0.6125,0>\right) & \left(s_{0},<1,0,0>\right)\end{array}\right]$, where $T_{i j}^{(1)} \cdot s_{i j}^{(1)}=\frac{1}{2}\left(T_{i j}^{(0)} \cdot s_{i j}^{(0)}\right) \oplus \frac{1}{2}\left(T_{i j}^{*(0)} \cdot s_{i j}^{*(0)}\right),\left(1-I_{i j}^{(1)}\right) \cdot s_{i j}^{(1)}=\frac{1}{2}\left(\left(1-I_{i j}^{(0)}\right) \cdot s_{i j}^{(0)}\right) \oplus \frac{1}{2}\left(\left(1-I_{i j}^{*(0)}\right) \cdot s_{i j}^{*(0)}\right)$ and $\left(1-F_{i j}^{(1)}\right) \cdot s_{i j}^{(1)}=\frac{1}{2}\left(\left(1-F_{i j}^{(0)}\right) \cdot s_{i j}^{(0)}\right) \oplus \frac{1}{2}\left(\left(1-F_{i j}^{*(0)}\right) \cdot s_{i j}^{*(0)}\right)$. As $C X\left(K^{(1)}\right) \approx 0.9314>C X$, output $K^{(1)}$.

Step 3: Aggregate each row of preference values in $K^{(1)}(i \neq j)$ using SNLGM operator. Then the overall preferences are $p x_{1} \approx\left(s_{0.762},<0.5966,0.7132,0.6882>\right), p x_{2} \approx\left(s_{-0.3160},<0.5570,0.6943,0.7127>\right)$, $p x_{3} \approx\left(s_{-1.7106},<0.5039,0.5392,0.2710>\right)$ and $p x_{4} \approx\left(s_{0.1602},<0.5102,0.5307,0.3090>\right)$.

Step 4: Calculate the score function $S\left(p x_{i}\right)(i=1,2, \ldots, n)$ by Definition 7: $S\left(p x_{1}\right) \approx 0.2372, S\left(p x_{2}\right) \approx 0.1765$, $S\left(p x_{3}\right) \approx 0.1616$ and $S\left(p x_{4}\right) \approx 0.2896$.

Step 5: Because $S\left(p x_{4}\right)>S\left(p x_{1}\right)>S\left(p x_{2}\right)>S\left(p x_{3}\right) \Rightarrow x_{4}>x_{1}>x_{2}>x_{3}$, and the optimal alternative is $x^{*}=x_{4}$, the second alternative is $x_{1}$.

\subsection{Comparison Analysis}

Considering the concept of SNLPRs is newly proposed, several approaches related to other kinds of preference relations are chosen to make a comparison in this subsection.

As the expressions of basic elements in different preference relations are diverse, the first task is to transform the SNLNs in SNLPRs into the corresponding expression. Then, the same problem will be solved. The following are the information conversion process and major steps of the related methods:

(1) Single-valued trapezoidal neutrosophic preference relations (SVTNPRs) [44]

First, SNLNs in SNLPRs can be transformed into single-valued trapezoidal neutrosophic numbers (SVTNNs). A suitable way is changing the linguistic values of SNLNs into trapezoidal fuzzy numbers in SVTNNs and keeping membership degrees. The converted values can be denoted by $A_{i}=\left(a_{i}, b_{i}, c_{i}, d_{i}\right)=\left(\max \left\{\frac{2 i+2 g-1}{4 g+3}, 0\right\}, \frac{2 i+2 g}{4 g+3}, \frac{2 i+2 g+1}{4 g+3}, \min \left\{\frac{2 i+2 g+2}{4 g+3}, 1\right\}\right)$ according to [76]. As an illustration, $\left(s_{1},<0.7,0.5,0.4>\right)$ can be regarded as $([0.474,0.526,0.579,0.632],<$ $0.7,0.5,0.4>$ ). Then, the corresponding single-valued trapezoidal neutrosophic matrix 


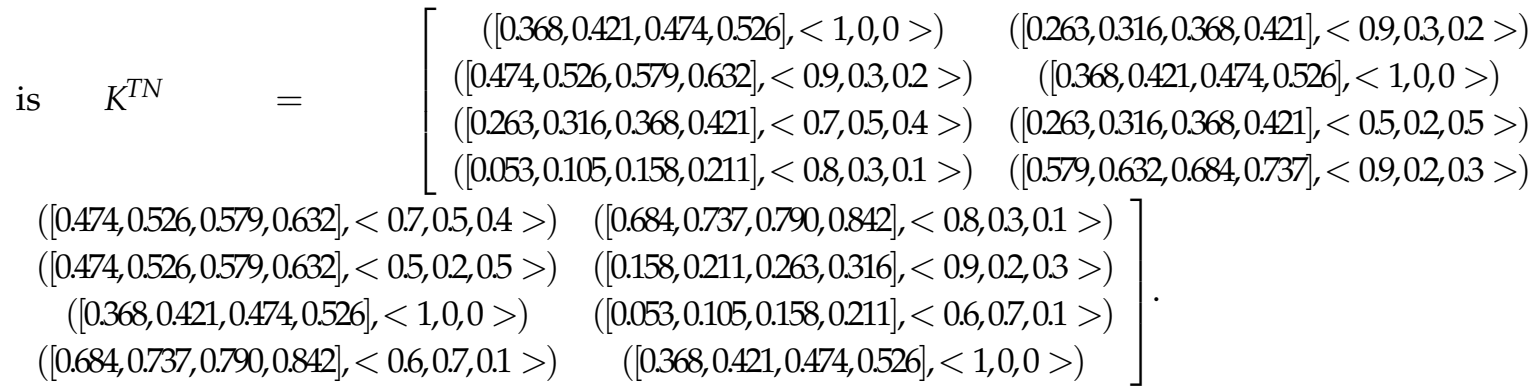

Subsequently, using the method in [44] to get the consistent preference matrix, and the ranking is $x_{4}>x_{1}>x_{2}>x_{3}$.

\section{(2) ILPRs [55]}

In the beginning, SNLNs should be converted into intuitionistic linguistic numbers. The linguistic values can remain, $\frac{1}{3}\left(T_{i j}+1-I_{i j}+1-F_{i j}\right)$ may be equivalent to $u$, and $v=1-u$. As an example, $\left(s_{-1},<\right.$ $0.9,0.3,0.2>$ ) can be converted into $\left\langle s_{-1},(0.8,0.2)>\right.$. Then, an intuitionistic linguistic preference relation

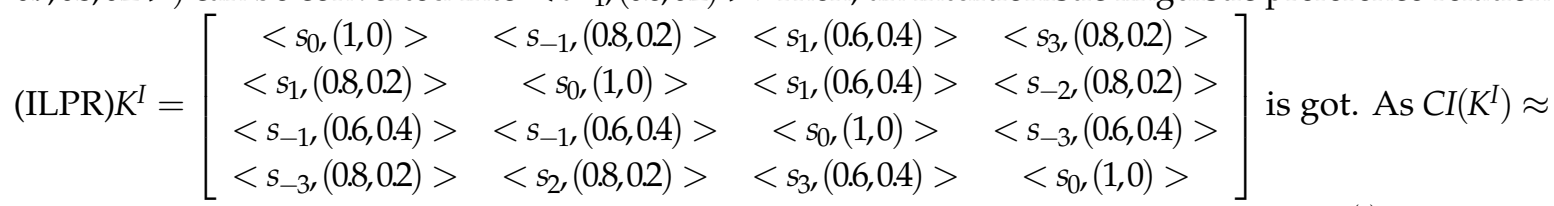
$0.8819<\theta(0.9016)$, let $\beta=0.8$, and then the consistency index of the adjusted ILPR is $C I\left(K^{I(1)}\right) \approx 0.9069>$ $\theta(0.9016)$. Thus, using the method in [55], the preferred degree matrix $U=\left(\begin{array}{cccc}1 / 2 & 1 & 1 & 6 / 7 \\ 0 & 1 / 2 & 1 & 0 \\ 0 & 0 & 1 / 2 & 0 \\ 1 / 7 & 1 & 1 & 1 / 2\end{array}\right)$. Since the preferred degrees $r_{1}\left(\frac{47}{14}\right)>r_{4}\left(\frac{37}{14}\right)>r_{2}\left(\frac{3}{2}\right)>r_{3}\left(\frac{1}{2}\right)$, then $x_{1} \succ x_{4} \succ x_{2} \succ x_{3}$.

\section{(3) HFLPRs [54]}

Firstly, SNLNs can be converted to hesitant fuzzy linguistic term sets. For example, $\left(s_{-} 0.9, s_{-0.3}, s_{-0.2}\right)$ can take the place of $\left(s_{-1},<0.9,0.3,0.2>\right)$. Hence, the corresponding HFLPR is $K^{H}=$ $\left[\begin{array}{cccc}\left(s_{0}\right) & \left(s_{-0.9}, s_{-0.3}, s_{-0.2}\right) & \left(s_{0.4}, s_{0.5}, s_{0.7}\right) & \left(s_{0.3}, s_{0.9}, s_{24}\right) \\ \left(s_{0.9}, s_{0.3}, s_{0.2}\right) & \left(s_{0}\right) & \left(s_{02}, s_{0.5}, s_{0.5}\right) & \left(s_{-1.8}, s_{-0.6}, s_{-0.4}\right) \\ \left(s_{-0.4}, s_{-0.5}, s_{-0.7}\right) & \left(s_{-02}, s_{-0.5}, s_{-0.5}\right) & \left(s_{0}\right) & \left(s_{-21}, s_{-1.8}, s_{-0.3}\right) \\ \left(s_{-0.3}, s_{-0.9}, s_{-24}\right) & \left(s_{1.8}, s_{0.6}, s_{0.4}\right) & \left(s_{21}, s_{1.8}, s_{0.3}\right) & \left(s_{0}\right)\end{array}\right]$. The expected 2-tuple linguistic preference relation is $E^{H(1)}=\left[\begin{array}{cccc}\left(s_{0}, 0\right) & \left(s_{0},-7 / 15\right) & \left(s_{1},-7 / 15\right) & \left(s_{1}, 1 / 5\right) \\ \left(s_{0}, 7 / 15\right) & \left(s_{0}, 0\right) & \left(s_{0}, 2 / 5\right) & \left(s_{-1}, 1 / 15\right) \\ \left(s_{-1}, 7 / 15\right) & \left(s_{0},-2 / 5\right) & \left(s_{0}, 0\right) & \left(s_{-1},-2 / 5\right) \\ \left(s_{-1},-1 / 5\right) & \left(s_{1},-1 / 15\right) & \left(s_{1}, 2 / 5\right) & \left(s_{0}, 0\right)\end{array}\right]$. Because $C I\left(E^{H(1)}\right) \approx$ $0.0860<\overline{C I}(0.1347)$, the matrix is acceptably consistent. Then use the aggregation operators and comparison method, and the ranking is $x_{1} \succ x_{4} \succ x_{2} \succ x_{3}$.

(4) LPRs [77]

At first, the conversion function $k_{i j}^{L}=\frac{1}{3}\left(T_{i j}+1-I_{i j}+1-F_{i j}\right) k_{i j}$ will be used to convert SNLNs in SNLPR $K$ into linguistic variables in a LPR $K^{L}$. For instance, $\left(s_{-1},<0.9,0.3,0.2>\right)$ can be replaced by $s_{-0.8}$. Then $K^{L}=\left[\begin{array}{cccc}s_{0} & s_{-0.8} & s_{0.6} & s_{24} \\ s_{0.8} & s_{0} & s_{0.6} & s_{-1.6} \\ s_{-0.6} & s_{-0.6} & s_{0} & s_{-1.8} \\ s_{-24} & s_{1.6} & s_{1.8} & s_{0}\end{array}\right]$. Since $C I\left(K^{L}\right) \approx 0.2667>\delta_{0}(0.1347)$, the automatic iterative Algorithm 1 in [77] is used. The consistency index of the adjusted LPR is $C I\left(K^{L(1)}\right) \approx 0.1334<\delta_{0}(0.1347)$, and then the final ranking is $x_{4}>x_{1}>x_{2}>x_{3}$. 
Subsequently, comparisons with each method in terms of backgrounds, consistency-improving processes, ranking methods, and ranking results are made in Table 2.

Table 2. Comparison of different methods.

\begin{tabular}{ccccc}
\hline Approaches & Backgrounds & Improving Consistency & Ranking Methods & Ranking Orders \\
\hline Liang et al. [44] & SVTNPRs & Interactive feedback & Arithmetic operator & $x_{4}>x_{1}>x_{2}>x_{3}$ \\
Meng et al. [55] & ILPRs & Automatic iteration & Preferred degrees & $x_{1} \succ x_{4} \succ x_{2} \succ x_{3}$ \\
Wu and Xu [54] & HFLPRs & Interactive feedback & Expected values & $x_{1} \succ x_{4} \succ x_{2} \succ x_{3}$ \\
Jin et al. [77] & LPRs & Automatic iteration & Arithmetic operator & $x_{4}>x_{1}>x_{2}>x_{3}$ \\
The proposed method & SNLPRs & Automatic iteration & Geometric operator & $x_{4}>x_{1}>x_{2}>x_{3}$ \\
\hline
\end{tabular}

In the ranking results of Table 2 only the order of $x_{1}$ and $x_{4}$ varies, which demonstrates the effectiveness of the proposed method. The in-depth comparison analyses are shown as follows:

(1) Comparison with References [44] and [77]: the same ranking results are obtained using the methods in $[44,77]$ and our approach. An interactive feedback is used to improve the consistency in [44]. It may be a little difficult for DMs to do this work, especially when the alternatives are numerous. In addition, the arithmetic operator used may cause a reversal of ranking in some cases. Jin et al. described information with linguistic term sets in Reference [77]. However, all of the membership degrees are missing in LPRs. And the arithmetic operator used in Reference [77] also has the limitation of sorting reversal.

(2) Comparison with Reference [55]: both [55] and our method choose the automatic iteration to improve consistency. The reason for the different ranking results may be that there are only membership and non-membership degrees in ILPRs. The conversion function possibly led to a loss of the original information.

(3) Comparison with Reference [54]: the difference between [54] and our approach is that there is no process of consistency improvement in HFLPRs. Moreover, the truth-membership, indeterminacy-membership, and false-membership of the linguistic values in SNLPRs have identical roles in HFLPRs. This may be another explanation of the different rankings.

According to the analysis above, the strengths of the presented approach are obvious. First of all, the basic elements in SNLPRs, SNLNs, contain three independent membership degrees to describe the consistent, hesitant, and inconsistent information, respectively. It means that the problem of evaluation information being missing is avoided to a greater extent. Thus, the proposed method is more suitable for solving problems in a simplified neutrosophic linguistic environment. Secondly, the consistency-improving process is an automatic iteration algorithm. It saves time and increases convenience for DMs. In addition, as mentioned in Section 4.4, the geometric operator being used may avoid the problem of ranking reversal. It is easy for us to understand and operate. Finally, the flexibility is increased as the linguistic scale function can be changed in different semantic situations.

\section{Discussion and Conclusions}

Appropriate project delivery systems play an irreplaceable role in promoting the development of the construction industry. The paper provided a decision-making approach with SNLPRs to solve the problem of selecting an optimal system under simplified neutrosophic linguistic circumstances. Several distance measures of SNLNs, which are the basic elements of SNLPRs, were redefined. They can overcome the drawbacks of the definition of Ye [70], so that the differences between two SNLNs can be well distinguished. Moreover, the paper created a distance-based consistency index to check the consistency of SNLPRs. A consistency-improving algorithm was also suggested. The effectiveness and advantages of this method were displayed by an illustration of selecting project delivery systems and the corresponding comparison analysis. 
Nevertheless, the proposed method still has some limitations, such as a case of SNLPRs with incomplete assessment information. In order to make the method based on SNLPRs more effectively and widely used in engineering projects, several future works can be planned as follow: (1) Other kinds of consistency, such as the multiplicative consistency of SNLPRs may be presented; (2) a situation where the linguistic term sets are unbalanced [78] may be under consideration; (3) decision-making methods based on incomplete SNLPRs are worth studying.

Acknowledgments: The authors thank the editors and anonymous reviewers for their helpful comments and suggestions. This work was supported by a Key Project of Hunan Social Science Achievement Evaluation Committee (XSP2016040508), the human philosophy social science fund project (15JD21), and the Natural Science Foundation of Hunan Province (2017JJ3181).

Author Contributions: Sui-Zhi Luo, Peng-Fei Cheng, and Jian-Qiang Wang conceived and worked together to achieve this work, Sui-Zhi Luo wrote the paper; Yuan-Ji Huang made contributions to the case study.

Conflicts of Interest: The authors declare no conflict of interest.

\section{References}

1. Uhlik, F.T.; Eller, M.D. Alternative delivery approaches for military medical construction projects. J. Archit. Eng. 1996, 5, 149-155. [CrossRef]

2. Partington, D.; Pellegrinelli, S.; Young, M. Attributes and levels of programme management competence: An interpretive study. Int. J. Proj. Manag. 2005, 23, 87-95. [CrossRef]

3. Hong, K.E.; Liu, C.; Management, S.O. Research on importance ranking of influencing factors on selecting project delivery system. J. Civ. Eng. Manag. 2014, 3, 2224-2230.

4. Lahdenperä, P.; Koppinen, T. Financial analysis of road project delivery systems. J. Financ. Manag. Prop. Constr. 2009, 14, 61-78. [CrossRef]

5. Mostafavi, A.; Karamouz, M. Selecting appropriate project delivery system: Fuzzy approach with risk analysis. J. Constr. Eng. Manag. 2010, 136, 923-930. [CrossRef]

6. Wang, L.Y.; An, X.W.; Li, H.M. Applying fuzzy set model for selecting project delivery system. In Proceedings of the International Conference on Simulation and Modeling Methodologies, Technologies and Applications, Vienna, Austria, 28-30 August 2014; Volume 60, pp. 1301-1308.

7. Barati, K.; Shiran, G.R.; Sepasgozar, S.M.E. Selecting optimal project delivery system for infrastructural projects using analytic hierarchy process. Am. J. Civ. Eng. Archit. 2015, 3, 212-217.

8. Mahdi, I.M.; Alreshaid, K. Decision support system for selecting the proper project delivery method using analytical hierarchy process (AHP). Int. J. Proj. Manag. 2005, 23, 564-572. [CrossRef]

9. Smarandache, F. A Unifying Field in Logics. Neutrosophy: Neutrosophic Probability, Set and Logic; American Research Press: Rehoboth, DE, USA, 1999.

10. Chen, J.Q.; Ye, J. Some single-valued neutrophic dombi weighted aggregation operators for multiple attribute decision-making. Symmetry 2017, 9, 82. [CrossRef]

11. Peng, H.G.; Zhang, H.Y.; Wang, J.Q. Probability multi-valued neutrosophic sets and its application in multi-criteria group decision-making problems. Neural Comput. Appl. 2016, 1-21. [CrossRef]

12. Ye, J. Trapezoidal neutrosophic set and its application to multiple attribute decision-making. Neural Comput. Appl. 2015, 26, 1157-1166. [CrossRef]

13. Ma, Y.X.; Wang, J.Q.; Wang, J.; Wu, X.H. An interval neutrosophic linguistic multi-criteria group decision-making method and its application in selecting medical treatment options. Neural Comput. Appl. 2017, 28, 2745-2764. [CrossRef]

14. Li, Y.Y.; Zhang, H.Y.; Wang, J.Q. Linguistic neutrosophic sets and their application in multi-criteria decision-making problem. Int. J. Uncertain. Quantif. 2017, 7, 135-154. [CrossRef]

15. Tian, Z.P.; Wang, J.; Wang, J.Q.; Zhang, H.Y. An improved MULTIMOORA approach for multi-criteria decision-making based on interdependent inputs of simplified neutrosophic linguistic information. Neural Comput. Appl. 2016, 1-13. [CrossRef]

16. Peng, J.J.; Wang, J.Q.; Zhang, H.Y.; Chen, X.H. An outranking approach for multi-criteria decision-making problems with simplified neutrosophic sets. Appl. Soft Comput. 2014, 25, 336-346. [CrossRef] 
17. Zhang, H.Y.; Wang, J.Q.; Chen, X.H. An outranking approach for multi-criteria decision-making problems with interval-valued neutrosophic sets. Neural Comput. Appl. 2016, 27, 615-627. [CrossRef]

18. Zhang, M.C.; Liu, P.D.; Shi, L.L. An extended multiple attribute group decision-making TODIM method based on the neutrosophic numbers. J. Intell. Fuzzy Syst. 2016, 30, 1773-1781. [CrossRef]

19. Stanujkic, D.; Zavadskas, E.K.; Smarandache, F.; Brauers, W.K.M.; Karabasevic, D. A neutrosophic extension of the MULTIMOORA method. Informatica 2017, 28, 181-192. [CrossRef]

20. Peng, J.J.; Wang, J.Q.; Yang, W.E. A multi-valued neutrosophic qualitative flexible approach based on likelihood for multi-criteria decision-making problems. Int. J. Syst. Sci. 2017, 48, 425-435. [CrossRef]

21. Bausys, R.; Zavadskas, E.K. Multi-criteria decision making approach by VIKOR under interval neutrosophic set environment. Econ. Comput. Econ. Cybern. Stud. Res. 2015, 49, 33-48.

22. Pouresmaeil, H.; Shivanian, E.; Khorram, E.; Fathabadi, H.S. An extended method using TOPSIS and VIKOR for multiple attribute decision making with multiple decision makers and single valued neutrosophic numbers. Adv. Appl. Stat. 2017, 50, 261-292. [CrossRef]

23. Li, Y.H.; Liu, P.D.; Chen, Y.B. Some single valued neutrosophic number heronian mean operators and their application in multiple attribute group decision making. Informatica 2016, 27, 85-110. [CrossRef]

24. Ye, J. Multiple attribute decision-making method using correlation coefficients of normal neutrosophic Sets. Symmetry 2017, 9, 80. [CrossRef]

25. Ye, J. Correlation coefficients of interval neutrosophic hesitant fuzzy sets and its application in a multiple attribute decision making method. Informatica 2016, 27, 179-202. [CrossRef]

26. Zavadskas, E.K.; Baušys, R.; Lazauskas, M. Sustainable assessment of alternative sites for the construction of a waste incineration plant by applying waspas method with single-valued neutrosophic set. Sustainability 2015, 7, 15923-15936. [CrossRef]

27. Zavadskas, E.K.; Baušys, R.; Stanujkic, D.; Magdalinovic-Kalinovic, M. Selection of lead-zinc flotation circuit design by applying waspas method with single-valued neutrosophic set. Acta Montan. Slov. 2016, 21, 85-92.

28. Baušys, R.; Juodagalvienè, B. Garage location selection for residential house by WASPAS-SVNS method. J. Civ. Eng. Manag. 2017, 23, 421-429. [CrossRef]

29. Nie, R.; Wang, J.; Zhang, H. Solving solar-wind power station location problem using an extended weighted aggregated sum product assessment (WASPAS) technique with interval neutrosophic sets. Symmetry 2017, 9, 106. [CrossRef]

30. Bausys, R.; Zavadskas, E.K.; KAKLAUSKAS, A. Application of neutrosophic set to multi-criteria decision making by COPRAS. Econ. Comput. Econ. Cybern. Stud. Res. 2015, 49, 91-105.

31. Čereška, A.; Zavadskas, E.K.; Cavallaro, F.; Podvezko, V.; Tetsman, I.; Grinbergienè, I. Sustainable assessment of aerosol pollution decrease applying multiple attribute decision-making methods. Sustainability 2016, 8 , 586. [CrossRef]

32. Herrera-Viedma, E.; Herrera, F.; Chiclana, F.; Luque, M. Some issues on consistency of fuzzy preference relations. Eur. J. Oper. Res. 2004, 154, 98-109. [CrossRef]

33. Xu, Y.J.; Herrera, F.; Wang, H.M. A distance-based framework to deal with ordinal and additive inconsistencies for fuzzy reciprocal preference relations. Inf. Sci. 2016, 328, 189-205. [CrossRef]

34. Chiclana, F.; Herrera-Viedma, E.; Alonso, S.; Herrera, F. Cardinal consistency of reciprocal preference relations: A characterization of multiplicative transitivity. IEEE Trans. Fuzzy Syst. 2009, 17, 14-23. [CrossRef]

35. Meng, F.Y.; Chen, X.H.; Zhang, Y.L. Consistency-based linear programming models for generating the priority vector from interval fuzzy preference relations. Appl. Soft Comput. 2016, 41, 247-264. [CrossRef]

36. Dong, Y.C.; Li, C.C.; Chiclana, F.; Herrera-Viedma, E. Average-case consistency measurement and analysis of interval-valued reciprocal preference relations. Knowl. Based Syst. 2016, 114, 108-117. [CrossRef]

37. Wu, J.; Chiclana, F.; Liao, H.C. Isomorphic multiplicative transitivity for intuitionistic and interval-valued fuzzy preference relations and its application in deriving their priority vectors. IEEE Trans. Fuzzy Syst. 2016. [CrossRef]

38. Yang, Q.; Zhang, Z.S.; You, X.S.; Chen, T. Evaluation and classification of overseas talents in China based on the BWM for intuitionistic relations. Symmetry 2016, 8, 137. [CrossRef]

39. Xu, G.L.; Wan, S.P.; Wang, F.; Dong, J.Y.; Zeng, Y.F. Mathematical programming methods for consistency and consensus in group decision making with intuitionistic fuzzy preference relations. Knowl. Based Syst. 2016, 98, 30-43. [CrossRef] 
40. Wang, Z.J.; Tong, X. Consistency analysis and group decision making based on triangular fuzzy additive reciprocal preference relations. Inf. Sci. 2016, 361-362, 29-47. [CrossRef]

41. Liu, F.; Pedrycz, W.; Wang, Z.X.; Zhang, W.G. An axiomatic approach to approximation-consistency of triangular fuzzy reciprocal preference relations. Fuzzy Sets Syst. 2017, 322, 1-18. [CrossRef]

42. Zhu, B.; Xu, Z.S.; Xu, J.P. Deriving a ranking from hesitant fuzzy preference relations under group decision making. IEEE Trans. Cybern. 2014, 44, 1328. [CrossRef] [PubMed]

43. Xu, Y.J.; Cabrerizo, F.J.; Herrera-Viedma, E. A consensus model for hesitant fuzzy preference relations and its application in water allocation management. Appl. Soft Comput. 2017, 58, 265-284. [CrossRef]

44. Liang, R.X.; Wang, J.; Zhang, H.Y. A multi-criteria decision-making method based on single-valued trapezoidal neutrosophic preference relations with complete weight information. Neural Comput. Appl. 2017, 1-16. [CrossRef]

45. Yang, Y.; Hu, J.H.; An, Q.X.; Chen, X.H. Group decision making with multiplicative triangular hesitant fuzzy preference relations and cooperative games method. Int. J. Uncertain. Quantif. 2017, 7, 271-284. [CrossRef]

46. Wang, C.; Wang, J. A multi-criteria decision-making method based on triangular intuitionistic fuzzy preference information. Intell. Autom. Soft Comput. 2016, 22, 473-482. [CrossRef]

47. Herrera, F.; Herrera-Viedma, E. Choice functions and mechanisms for linguistic preference relations. Eur. J. Oper. Res. 2000, 120, 144-161. [CrossRef]

48. Nie, R.; Wang, J.; Li, L. A shareholder voting method for proxy advisory firm selection based on 2-tuple linguistic picture preference relation. App. Soft Comput. 2017, 60, 520-539. [CrossRef]

49. Xu, Y.J.; Wei, C.P.; Sun, H. Distance-based nonlinear programming models to identify and adjust inconsistencies for linguistic preference relations. Soft Comput. 2017, 1-17. [CrossRef]

50. Zhang, G.Q.; Dong, Y.C.; Xu, Y.F. Consistency and consensus measures for linguistic preference relations based on distribution assessments. Inf. Fusion 2014, 17, 46-55. [CrossRef]

51. Zhang, X.; Wang, J. Consensus-based framework to MCGDM under multi-granular uncertain linguistic environment. J. Intell. Fuzzy Syst. 2017, 33, 1263-1274. [CrossRef]

52. Wang, J.; Wang, J.Q.; Tian, Z.; Zhao, D. A multi-hesitant fuzzy linguistic multi-criteria decision-making approach for logistics outsourcing with incomplete information. Int. Trans. Oper. Res. 2017. [CrossRef]

53. Wang, H.; Xu, Z.S. Some consistency measures of extended hesitant fuzzy linguistic preference relations. Inf. Sci. 2015, 297, 316-331. [CrossRef]

54. Wu, Z.B.; Xu, J.P. Managing consistency and consensus in group decision making with hesitant fuzzy linguistic preference relations. Omega 2016, 65, 28-40. [CrossRef]

55. Meng, F.Y.; Tang, J.; An, Q.X.; Chen, X.H. Decision making with intuitionistic linguistic preference relations. Int. Trans. Oper. Res. 2017. [CrossRef]

56. Nie, R.; Wang, J.; Li, L. 2-tuple linguistic intuitionistic preference relation and its application in sustainable location planning voting system. J. Intell. Fuzzy Syst. 2017, 33, 885-899. [CrossRef]

57. Zhang, Y.; Ma, H.X.; Liu, B.H.; Liu, J. Group decision making with 2-tuple intuitionistic fuzzy linguistic preference relations. Soft Comput. 2012, 16, 1439-1446. [CrossRef]

58. Zhang, Y.X.; Xu, Z.S.; Wang, H.; Liao, H.C. Consistency-based risk assessment with probabilistic linguistic preference relation. Appl. Soft Comput. 2016, 49, 817-833. [CrossRef]

59. Peng, J.; Wang, J.; Yang, L.; Qian, J. A novel multi-criteria group decision-making approach using simplified neutrosophic information. Int. J. Uncertain. Quantif. 2017. [CrossRef]

60. Wang, J.Q.; Yang, Y.; Li, L. Multi-criteria decision-making method based on single valued neutrosophic linguistic Maclaurin symmetric mean operators. Neural Comput. Appl. 2016, 1-19. [CrossRef]

61. Wu, X.; Wang, J. Cross-entropy measures of multi-valued neutrosophic sets and its application in selecting middle-level manager. Int. J. Uncertain. Quantif. 2017, 7, 155-176. [CrossRef]

62. Tian, Z.P.; Wang, J.; Zhang, H.Y.; Wang, J.Q. Multi-criteria decision-making based on generalized prioritized aggregation operators under simplified neutrosophic uncertain linguistic environment. Int. J. Mach. Learn. Cybern. 2016, 1-17. [CrossRef]

63. Yu, S.; Zhang, H.; Wang, J. Hesitant fuzzy linguistic maclaurin symmetric mean operators and their applications to multi-criteria decision-making problem. Int. J. Intell. Syst. 2017. [CrossRef]

64. Wang, J.; Wang, J.Q.; Zhang, H.Y.; Chen, X.H. Distance-based multi-criteria group decision-making approaches with multi-hesitant fuzzy linguistic information. Int. J. Inf. Technol. Decis. Mak. 2017, 16, 1069-1099. [CrossRef] 
65. Zhang, H.Y.; Peng, H.G.; Wang, J.; Wang, J.Q. An extended outranking approach for multi-criteria decision-making problems with linguistic intuitionistic fuzzy numbers. Appl. Soft Comput. 2017, 59, $462-474$. [CrossRef]

66. Xu, Z.S. Deviation measures of linguistic preference relations in group decision making. Omega 2005, 33, 249-254. [CrossRef]

67. Zadeh, L.A. The concept of a linguistic variable and its application to approximate reasoning. Inf. Sci. 1975, 8, 199-249. [CrossRef]

68. Wang, J.Q.; Wu, J.T.; Wang, J.; Zhang, H.Y.; Chen, X.H. Interval-valued hesitant fuzzy linguistic sets and their applications in multi-criteria decision-making problems. Inf. Sci. 2014, 288, 55-72. [CrossRef]

69. Tian, Z.P.; Wang, J.; Zhang, H.Y.; Chen, X.H.; Wang, J.Q. Simplified neutrosophic linguistic normalized weighted Bonferroni mean operator and its application to multi-criteria decision-making problems. FILOMAT 2016, 30, 3339-3360. [CrossRef]

70. Ye, J. An extended TOPSIS method for multiple attribute group decision making based on single valued neutrosophic linguistic numbers. J. Intell. Fuzzy Syst. 2015, 28, 247-255.

71. Tian, Z.P.; Wang, J.; Wang, J.Q.; Zhang, H.Y. Simplified neutrosophic linguistic multi-criteria group decision-making approach for green product development. Group Decis. Negot. 2017, 26, 597-627. [CrossRef]

72. Zhou, L.G.; Zhou, Y.Y.; Liu, X.; Chen, H.Y. Some ILOWA operators and their applications to group decision making with additive linguistic preference relations. J. Intell. Fuzzy Syst. 2015, 29, 831-843. [CrossRef]

73. Dong, Y.C.; Xu, Y.; Li, H. On consistency measures of linguistic preference relations. Eur. J. Oper. Res. 2008, 189, 430-444. [CrossRef]

74. Zhu, B.; Xu, Z.S. Consistency measures for hesitant fuzzy linguistic preference relations. IEEE Trans. Fuzzy Syst. 2014, 22, 35-45. [CrossRef]

75. Hou, F.J. A hierarchical decision model based on pairwise comparisons. Fundam. Inform. 2016, 144, $333-348$. [CrossRef]

76. Zhang, Z.; Guo, C.H. A method for multi-granularity uncertain linguistic group decision making with incomplete weight information. Knowl. Based Syst. 2012, 26, 111-119. [CrossRef]

77. Jin, F.F.; Ni, Z.W.; Chen, H.Y.; Li, Y.P. Approaches to decision making with linguistic preference relations based on additive consistency. Appl. Soft Comput. 2016, 49, 71-80. [CrossRef]

78. Cabrerizo, F.J.; Al-Hmouz, R.; Morfeq, A.; Balamash, A.S.; Martínez, M.A.; Herrera-Viedma, E. Soft consensus measures in group decision making using unbalanced fuzzy linguistic information. Soft Comput. 2017, 21, 3037-3050. [CrossRef] 